\title{
Temporal Coding of Periodicity Pitch in the Auditory System: An Overview
}

\author{
Peter Cariani \\ Eaton Peabody Laboratory, Massachusetts Eye and Ear Infirmary, Department of Otology and \\ Laryngology, Harvard Medical School, Boston, Massachusetts, USA
}

\section{SUMMARY}

This paper outlines a taxonomy of neural pulse codes and reviews neurophysiological evidence for interspike interval-based representations for pitch and timbre in the auditory nerve and cochlear nucleus. Neural pulse codes can be divided into channel-based codes, temporal-pattern codes, and time-of-arrival codes. Timings of discharges in auditory nerve fibers reflect the time structure of acoustic waveforms, such that the interspike intervals that are produced precisely convey information concerning stimulus periodicities. Population-wide inter-spike interval distributions are constructed by summing together intervals from the observed responses of many single Type $I$ auditory nerve fibers. Features in such distributions correspond closely with pitches that are heard by human listeners. The most common allorder interval present in the auditory nerve array almost invariably corresponds to the pitch frequency, whereas the relative fraction of pitchrelated intervals amongst all others qualitatively corresponds to the strength of the pitch. Consequently, many diverse aspects of pitch perception are explained in terms of such temporal representations. Similar stimulus-driven temporal discharge patterns are observed in major neuronal populations of the cochlear nucleus. Population-interval distributions constitute an alternative time-domain strategy for

\footnotetext{
Reprint address:

243 Charles St., Boston MA 02114 USA

fax: +1- (617) 720-4408;

email: peter@epl.meei.harvard.edu
}

representing sensory information that complements spatially organized sensory maps. Similar autocorrelation-like representations are possible in other sensory systems, in which neural discharges are time-locked to stimulus waveforms.

\section{KEYWORDS}

neural codes, interspike intervals, autocorrelation, phase-locking, temporal correlation, sensory coding, vowels, voice pitch, speech perception

\section{THE NEURAL CODING PROBLEM}

The neural coding problem, how populations of neurons represent and convey information through trains of spikes, is fundamental to our understanding how sensory systems function (Boring, 1942; Mountcastle, 1967; Perkell \& Bullock, 1968; Uttal, 1973; Wasserman, 1992; Cariani, 1995; Rieke et al., 1997; Richmond \& Gawne, 1998; Gerstner, 1999). The neural coding problem in perception involves mappings (Fig. 1) between stimulus, neural response, and percepts, whose biological basis can be approached from considerations of structure, function, and functional organization. These considerations involve different disciplines. Psychophysics seeks to understand the relation between stimulus and percept. Currently most neuroscience research is devoted to understanding the structure-function relationship of neurons on both the molecular and cellular levels. At the neural systems level, most current sensory neurophysiology focuses on understanding the relation between stimulus and neural response (system identification). Although 
a great deal is known about neural response properties at many levels of the auditory system, we do not yet have firm understandings of which particular response properties subserve the perceptions of auditory-form qualities, such as pitch, timbre, consonance, and phonetic identity. For auditory forms, solution of the neural coding problem entails identifying which aspects of the neural response are responsible for the perceptual detections, discriminations, and recognitions that can be realized by the system as a whole. In semiotic terms, neural responses shorn of their functional roles are signs, whereas neural codes and representations constitute those aspects of the neural responses that have particular functional, informational significance. In the auditory context, a major focus of such investigations is to find strong psychoneural correspondences between patterns of activity in auditory neurons and the auditory percepts that invariably accompany them. Once such correspondences are found, then one can posit possible neural processing strategies that can make use of such information and look in the auditory pathway for specific neural mechanisms that might subserve such processing. The ultimate goal of these efforts is to understand the biological design principles, the functional organization of the auditory system as an informational system, that are essential for its perceptual and cognitive capabilities. Neural codes, the manner in which sensory information is represented by the system, lie at the heart of this functional organization.

A number of biological and behavioral constraints narrow the search for viable candidate codes. Knowing how the system is constructed, how the elements behave and how they are interconnected, places strong constraints on how

A EXPERIMENTAL

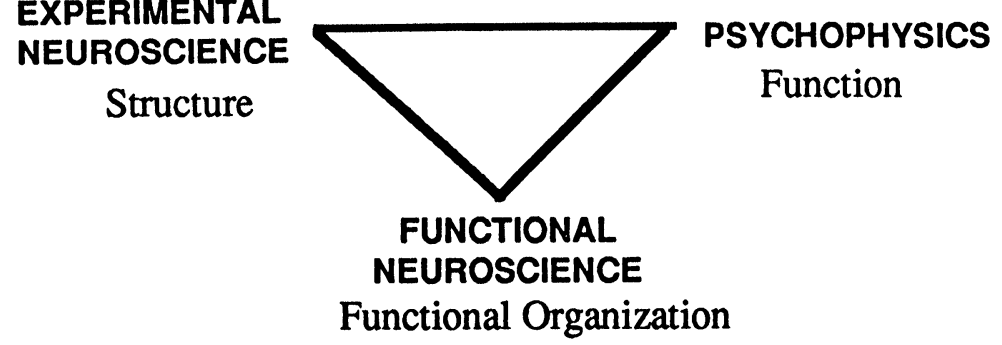

B

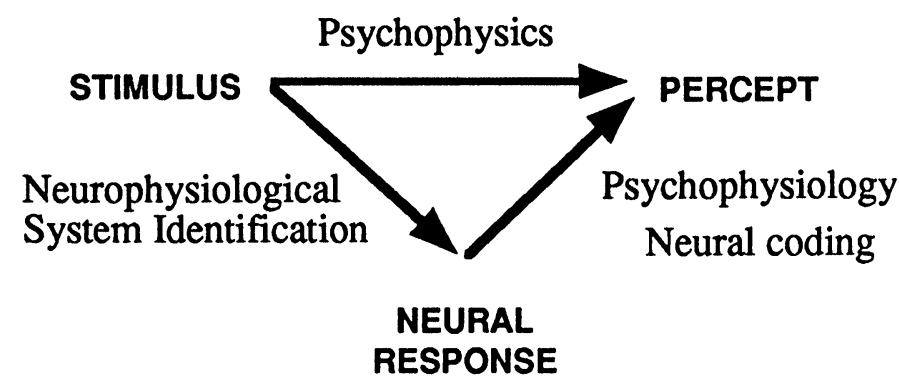

Fig. 1: Structure, function, and functional organization in the nervous system. Mappings between stimulus, neural responses, and their related percepts. 
the system can handle information. Neuroanatomy supplies us with the interconnections, neurophysiology with the response properties of the parts, and molecular and cellular neuroscience with more detailed understanding of their operation. Similarly, knowing what perceptual functions the system can and cannot perform imposes a different set of functional constraints. Here information-theoretic approaches have been used to quantify how much information about the stimulus can be extracted from neural responses under particular coding schemes (Bialek et al., 1991; Rieke et al., 1997; Richmond \& Gawne, 1998). Decision-theoretic approaches have been used to test how well neural information represented via particular coding schemes covaries with perceptual capabilities (Siebert, 1968; Siebert, 1970; Srulovicz \& Goldstein, 1977; Delgutte, 1995). For example, decisiontheoretic criteria can use the high precisions of perceptual discriminations that the system can perform under challenging conditions to narrow down the field of candidate codes. Potential codes are eliminated if not enough information exists in the neural response to support the observed precisions, or if the information is not present under all the confounding conditions under which the system is able to function. Strong perceptual and cognitive equivalence classes yield other clues as to the nature of the information being processed and of the modes by which it is utilized.

Neuroanatomical and neurophysiological considerations inform us as to how the parts of the system are interconnected and how they behave under particular conditions, but by themselves do not inform us as to which parts are essential for which functions, or how neural responses are interpreted by the rest of the system (Kiang, 1975). The psychological sciences inform us as to the functional capabilities of the system, but by themselves do not inform us the details of the neural mechanisms, what parts are needed, and how they must be organized to achieve perceptual functions. A complementary approach is therefore needed to focus on how the system is organized to perform its functions. Currently this approach comes under the rubric of functional, integrative, or computational neuroscience. In the context of informational functions, functional organization involves those aspects of neural responses that convey information and those aspects of neural structure that permit this information to inform behavior usefully.

Neuroanatomical and neurophysiological considerations inform us as to how the parts of the system are interconnected and how they behave under particular conditions, but by themselves they do not inform us as to which parts are essential for which functions - how neural responses are interpreted by the rest of the system (Kiang, 1975). The psychological sciences inform us as to the functional capabilities of the system, but by themselves they do not inform us the details of the neural mechanisms - what parts are needed and how they must be organized so as to achieve perceptual functions. A complementary approach is therefore needed to focus on how the system is organized so as to perform its functions. Currently this approach comes under the rubric of functional, integrative, or computational neuroscience. In the context of informational functions, functional organization involves those aspects of neural responses that convey information and those aspects of neural structure that permit this information to usefully inform behavior.

\section{TAXONOMY OF NEURAL PULSE CODES}

Many different kinds of neural pulse codes are possible (Fig. 2). Neural coding of sensory information can be based on discharge rates, interspike interval patterns, latency patterns, interneural discharge synchronies and correlations, spikeburst structure, or still more elaborate crossneuron volley-patterns. Sensory coding can be based on the mass-statistics of many independent neural responses (population codes) or on the joint properties of particular combinations of responses (ensemble codes) (Hatsopoulos et al., 1998). Amidst the many ways that neural spike trains can convey sensory information are 
fundamentally two basic ideas: "coding-bychannel" and "coding-by-time". Channel-based codes depend upon the activation of specific neural channels or of configurations of channels. Temporal codes, on the other hand, depend on the relative timings of neural discharges rather than on which particular neural channels respond how much. Temporal codes can be based on particular patterns of spikes within spike trains (temporalpattern codes) or on the relative times-of-arrivals of spikes (time-of-arrival codes).

The three different modes of neural coding: coding by channel, coding by temporal pattern, and coding by time-of-arrival, are complementary and correspond respectively to different, independent, and general aspects of signals:

a) the physical channel through which the signal is transmitted,

b) its internal form (for example, its waveform or Fourier spectrum), and

c) its time of arrival.
The absolute magnitude of the signal constitutes a fourth, intensive aspect that can be used in conjunction with the other three. For encoding multiple kinds of stimulus properties in a signaling system, each signal requires two independent variables, signal-type and signalvalue. One variable conveys the type or category of the information that is contained in the signal, whereas the other encodes the particular state of the signal amongst the possible alternative states. In artificial devices, the signal-type is most commonly conveyed by the particular channel through which a signal is sent (consider the many types of information conveyed by the respective wires leading to different gauges on the dashboard of a car). The identity of the channel conveys to the rest of the device what kind of information is being sent (namely, to which type of sensor the wire is connected). Similarly, in artifacts, signal-value is usually conveyed by the amplitude of the signal, often a voltage. Neural coding schemes similarly

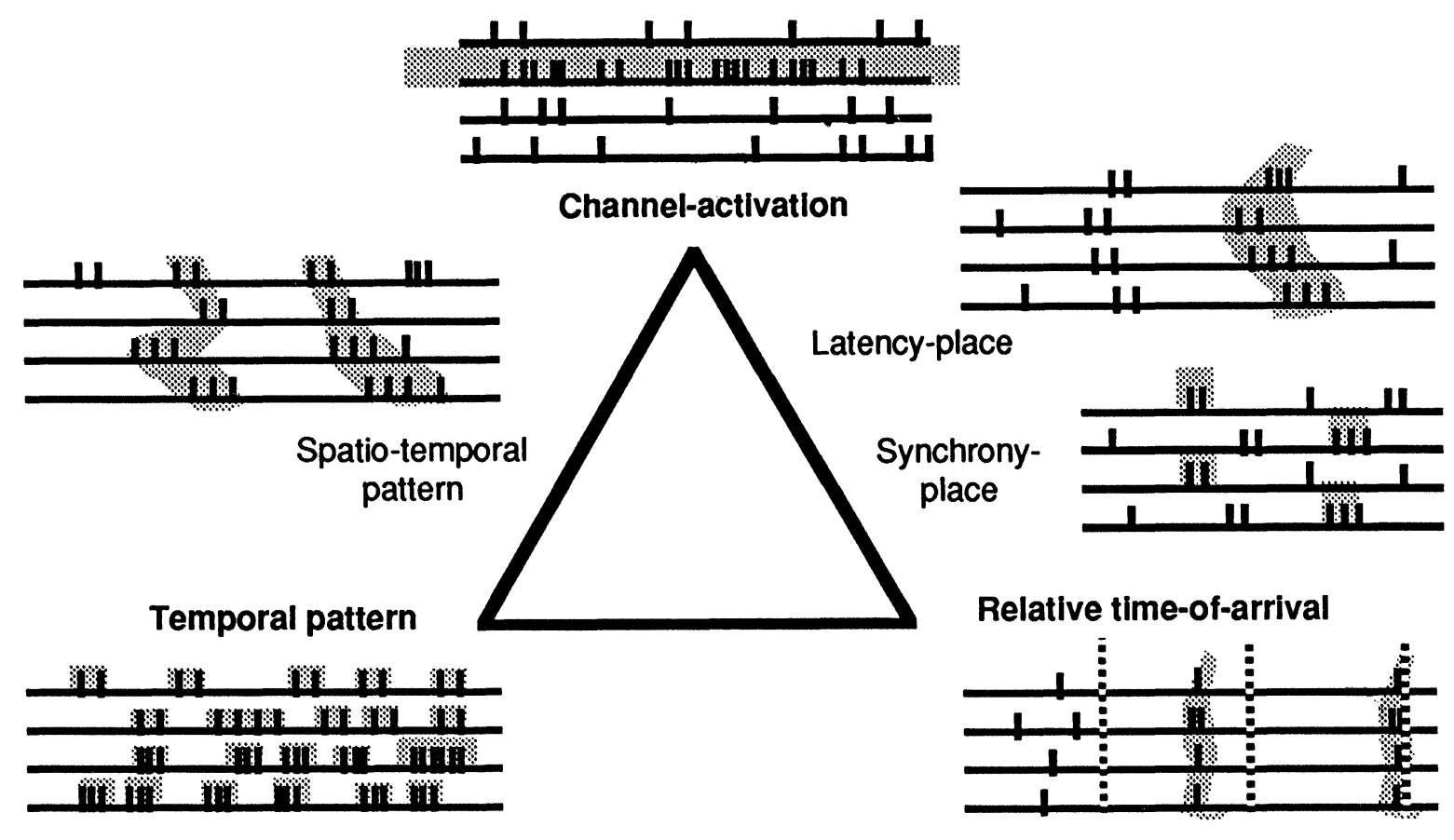

Fig. 2: A space of possible pulse codes. Three complementary modes of coding are shown at the vertices, with their combinations on the edges. 
require two sets of independent coding variables. Most typically, channel-based neural-coding schemes use the identities of neurons to encode signal-types, whereas some intensive measure of activation, such as discharge rate, encodes signalvalue. So constructed, channel-based coding schemes depend critically upon which particular neurons are activated how much. If the connectivities of neurons are suddenly rearranged in such a system, the coherence of neural representations will be disrupted, at least until the system can be adaptively rearranged to reflect the new channel-identities.

Many different channel-based coding schemes are possible. Such schemes can range from simple, unidimensional representations to lowdimensional sensory maps to higher dimensional feature detectors. In simple "doorbell" or "labeled line" systems, activation (or suppression) of a given neuron signals the presence or absence of one particular property. In more multipurpose schemes, neurons are differentially tuned to particular stimulus properties, such as frequency, periodicity, intensity, duration, or external location. Profiles of average discharge rates across a population of such tuned elements then convey multidimensional information about a stimulus. When spatially organized in a systematic manner by their tunings, these elements form sensory maps, in which spatial patterns of channel-activation can then represent arbitrary combinations of those stimulus properties. In lieu of coherent spatial order, tuned units can potentially convey their respective channel-identities through the specificity of spatially distributed neural connections. More complex constellations of properties can be represented via more complex concatenations of tunings to form highly specific "featuredetectors". In the absence of coherent tunings, combinations of idiosyncratic response properties can potentially form "across-neuron pattern codes" of the sort that are commonly proposed for the olfactory system. Nevertheless, idiosyncratic across-neuron patterns and associative learning mechanisms have fundamental difficulties in explaining common strong perceptual equivalence classes that are shared by most humans and are largely independent of an individual's particular history (Gesteland et al., 1965). Although these various functional organizations, from labeled lines to feature detectors to across-neuron patterns, encompass widely diverse modes of neural representation, all draw on the same basic strategy of coding-bychannel. In channel-coding schemes, it is usually further assumed that distinctions between alternative signal-states are encoded by different average discharge rates (Shadlen \& Newsome, 1994; Shadlen \& Newsome, 1998). The combination of channel- and rate-based coding has remained by far the dominant neural coding assumption throughout the history of neurophysiology (Boring, 1942; Barlow, 1995), and, consequently, forms the basis for nearly all our existing neural-network models.

Within channel-coding schemes, aspects of the neural response other than rate, such as relative latency or temporal pattern, can also play the role of encoding alternative signal states (for example, the latency-place and spatiotemporal codes shown in Fig. 2). In a simple latency-channel code, channels producing spikes at shorter latencies relative to the onset of a stimulus indicate stronger activation of tuned elements, which can be used to encode stimulus intensity (Stevens, 1971), location (Brugge et al., 1996), or other qualities. Common-response latency, in the form of interchannel synchrony, has been proposed as a strategy for grouping channels to form discrete, separate objects (Singer, 1990; Singer, 1995). In this scheme, rate patterns across simultaneously activated channels encode objectqualities, whereas interchannel synchronies (joint properties of response latencies) create perceptual organization, which channels combine to encode which objects. The concurrent use of multiple coding vehicles, channel, rate, and common time-of-arrival permits time-division multiplexing of multiple objects. Still, other kinds of asynchronous multiplexing are possible if other coding variables, such as common temporal pattern and phase coherence, are used (Cariani, 1997).

Characteristic temporal discharge patterns can also convey information about stimulus qualities. 
Neural codes that rely predominantly on the timings of neural discharges have been found in a variety of sensory systems (Mountcastle, 1967; Perkell \& Bullock, 1968; Chung et al., 1970; Kozak \& Reitboeck, 1974; Covey, 1980; Emmers, 1981; Bialek et al., 1991; Carr, 1993; Cariani, 1995; Cariani, 1997). Conceptually, these temporal codes can be divided into time-of-arrival and temporalpattern codes.

Time-of-arrival codes use the relative times of arrival of spikes in different channels to convey information about the stimulus. Examples of timeof-arrival codes are found in many sensory systems that utilize the differential times of arrival of stimuli at different receptor surfaces to infer the location of external objects (Bower, 1974; Carr, 1993). Strong examples are auditory localizations that rely on the time-of-arrival differences of acoustic signals at the two ears, echolocation range-findings that rely on time-of-arrival differences between emitted calls and their echoes, and electroceptive localizations that use the phase-differences of internally generated weak electric fields at different locations of the body to infer the presence of external phase distortions caused by nearby objects.

Temporal pattern codes, such as interspike interval codes, use temporal patterns between spikes to convey sensory information. In a temporal pattern code, the internal patterns of spike arrivals bear stimulus-related information. The simplest temporal pattern codes are interspike interval codes, in which stimulus periodicities are represented using the times between spike arrivals. More complex temporal pattern codes use higher-order time patterns consisting of interval sequences (Emmers, 1981; Lestienne \& Strehler, 1987). Like time-of-arrival codes, interval and interval-sequence codes could be called correlational codes because they rely on temporal correlations between individual spikearrival events. These codes should be contrasted with conceptions of temporal coding that rely on temporal variations in average discharge rate or discharge probability (for example, Richmond \& Gawne, 1998), which count numbers of events across stimulus presentations as a function of time and then perform a coarse temporal analysis on event-rates. Both time-of-arrival and temporalpattern codes depend on the stimulus impressing itself, in one way or another, on the timings of neural discharges. The stimulus-related temporal discharge patterns, on which temporal-pattern codes depend, can arise in two ways, through stimulus-locking and through intrinsic-time courses of response.

For stimulus-locking, the discharges of sensory neurons follow the time-amplitude course of the stimulus waveform. The highly stimuluslocked nature of discharges in the auditory nerve and the cochlear nucleus is evident in the peristimulus time histograms shown in the figures below. As long as a monotonic relation exists between the amplitude of the driving input and the probability of subsequent discharge, temporal correlations will be produced between waveform and spike train. In the auditory system, as in many other sensory systems, receptor cells depolarize when stereocilia are deflected in a particular direction, such that the timings of spikes predominantly occur during one phase of the stimulus waveform as it presents itself to the individual receptor (for example, after having been mechanically filtered by the cochlea). This form of stimulus-locking is known as phaselocking. In the auditory system, depending upon the species, strong phase-locking can exist up to several $\mathrm{kHz}$, dramatically declining as progressively higher frequencies are reached. Such phase-locking is also found in many other sensory systems, albeit usually at much lower frequencies. Phase-locked responses exist

a) to flutter-vibrations of the skin in mechanoception (Mountcastle, 1993),

b) to accelerations in the vestibular system,

c) to drifting gratings and flickering lights in the visual system (Pollen et al., 1989),

d) to inhalation cycles and odor pulses in olfaction (Macrides \& Chorover, 1972; Onoda \& Mori, 1980; Marion-Poll \& Tobin, 1992),

e) to self-produced electrical oscillations in electroception, and

f) to the movements of muscles in proprioceptive stretch receptors. 
A generalization can be made that every sensory system will show phase-locked responses to its adequate stimulus, provided that the contrast is sufficient to create distinguishable, phasedependent responses and that modulations are slow enough for phase-dependent responses to be separated temporally. To the extent that phaselocking exists, then the time intervals between the spikes that are produced (interspike intervals) reflect stimulus periodicities, such that time intervals themselves can serve as neural representations of stimulus form. In addition, phase-locked discharges register the arrival times of nonperiodic, transient, and unitary events, such that comparisons of the arrival times of the same event at different sensory surfaces (for example, the differential time-of-arrival of an acoustic wavefront at the two ears) can serve as neural representations of stimulus location relative to those sensory surfaces.

The intrinsic temporal patterns of neural response can also convey information concerning stimulus qualities. Such temporal response patterns can be characteristic of particular receptor types, individual neurons, local neural circuits, or even whole neural populations. Stimulus-related temporal discharge patterns that are not directly locked to the time structure of the stimulus have been observed in many sensory systems: olfaction (Kauer, 1974; Macrides, 1977; Marion-Poll \& Tobin, 1992; Laurent \& Naraghi, 1994); gustation (Covey, 1980; Di Lorenzo \& Hecht, 1993); spatial vision (Richmond et al., 1987; Richmond \& Gawne, 1998); color (Kozak \& Reitboeck, 1974; Wasserman, 1992). In some sensory modalities, temporal patterns of electrical stimulation appear to produce particular sensory qualities, such as taste and color (Young, 1977; Covey, 1980; Di Lorenzo \& Hecht, 1993), suggesting that the temporal patterns themselves may be the neural-coding vehicles that subserve these particular qualities. Stimulus-triggered intrinsic temporal patterns that are associated with conditioning and perceptual expectations have also been found in cortical regions (John \& Schwartz, 1978; John, 1990).
How might such intrinsic time patterns represent combinations of stimulus properties? One possibility is that the relative occurrence of different time patterns, associated with characteristic impulseor step-responses of particular neurons, can serve as markers that indicate the activation of particular subpopulations of neurons. Mixtures of odorants, tastants, and wavelengths of light would then produce mixtures of the respective temporal spike patterns of the receptors and neurons that they preferentially activate. As in the population-interval representation for pitch discussed below, patterns that are associated with the individual constituents, their interactions, and their fusions presumably would exist in the population time structure. These features could then be used to discriminate basic stimulus properties and to represent mixtures.

For different stimulus-receptor combinations, many ionic and molecular mechanisms in sensory receptors are available to produce differential kinetics of activation, inactivation, and recovery. In neural populations, temporal dynamics of excitation and inhibition could similarly produce characteristic temporal patterns. Both stimuluslocked and stimulus-triggered intrinsic temporal response patterns can be found throughout the auditory pathway. Extrinsic stimulus-locked patterns are most apparent in the lower stations, whereas intrinsic patterns become more apparent as one progresses to more central stations, where neural responses become increasingly dominated by the recent history of the system as a whole.

Finally, yet another dimension to neural codes involves the joint response properties of multiple neurons. This dimension is the distinction between population codes and ensemble codes (Deadyler \& Hampson, 1995; Hatsopoulos et al., 1998), between statistical orders and switchboards (John, 1972). To represent information, population codes use the mass statistics of stimulus-driven response properties of individual, largely independent units. Examples of such population codes are population-rate vectors in the motor cortex (Lee et al., 1998), or the auditory population-interval distributions presented below. In population codes, interdependencies between 
the responses of particular neurons are themselves irrelevant to the representation. Ensemble codes, on the other hand, use these interdependencies rather than common, stimulus-driven statistical structure to represent information. Response interdependencies can be reliably produced by specific interneural connectivities and timedelays. The resulting stimulus-related intrinsic correlations between the neuronal channels that are activated and/or synchronized, as well as between the latencies of spikes produced by different neurons, then can convey information about a stimulus. Perceptual grouping by means of channel-synchronizations that are not stimulusdriven would be an example of an ensemble code, in which statistics of channel activations by themselves would be insufficient for its interpretation; one would have to know which combinations of channels are synchronized at each moment. In the context of sensory coding, the relative merits of the stimulus-driven, massstatistics of population-codes versus the stimulustriggered, joint-response properties of ensemble codes remain to be more fully explored.

\section{THE NEURAL BASIS OF PITCH PERCEPTION}

The nature of the neural codes that subserve auditory perception have generated lively ongoing discussion and debate for most of the last 150 years (Boring, 1942; Wever, 1949; de Boer, 1976). For the most part, this discussion has been focused on whether frequency is represented (a) via rate-place codes, namely, neural dischargerate profiles in auditory frequency maps, or (b) via temporal codes, namely, interspike interval distributions (Siebert, 1970; Moore, 1997; Evans, 1978s). In many debates about neural coding, pitch has played a pivotal role mainly because pitch is a perceptual correlate of frequency (Boring, 1942; de Boer, 1976). At the same time, pitch is also a perceptual correlate of periodic waveforms, whether single pure tones or complexes consisting of many harmonically related partials. Operationally, pitch is defined as the frequency of a pure tone to which a given sound can be reliably matched. The percept provides a very rich test bed for understanding many aspects of perception. Like color, pitch is metameric; the same pitch can be evoked by many different stimuli that can differ markedly in their power spectra. When harmonically related partials are sounded together, strong pitches at their common, fundamental frequency (F0) can be produced in the absence of any spectral component at that frequency (specifically, the "missing fundamental" is heard). These pitches are often called "low" pitches because the fundamental has a lower pitch than those that are associated with any of the individual partials. Such pitches are often called "periodicity" pitches because the low pitch at the fundamental reflects the periodicity of the recurring time pattern that is associated with the whole harmonic complex. Thus, combinations of partials give rise to new low pitches that are not heard in the separate constituents. Pitches produced by such complex tones are consequently "emergent" perceptual Gestalts, products of the relations between parts rather than of the parts themselves. Finally, pitch is largely invariant with respect to a host of factors, such as stimulus intensity and location, that produce large changes in the responses of auditory neurons. These perceptual invariances focus the search for the neural basis of pitch on the aspects of neural activity displaying similar stability.

Historically, a strong case for temporal coding of pitch has always been made ( Troland, 1929; Boring, 1942; Wever, 1949), although the pendulum of scientific opinion has swung back and forth between spectral pattern and temporal theories several times now (de Boer, 1976; Evans, 1978; Lyon \& Shamma, 1995). Although autocorrelation-based models for pitch were first proposed almost a half-century ago (Licklider, 1951; Licklider, 1959), only during the last two decades have similar kinds of global, intervalbased models been revived and extended (van Noorden, 1982; de Cheveigné, 1986; Meddis \& Hewitt, 1991; Slaney \& Lyon, 1993; Lyon \& Shamma, 1995; Cariani \& Delgutte, 1996a, 1996b; Meddis \& O'Mard, 1997; Moore, 1997). 
In physiological studies at the level of the auditory nerve of the cat (Cariani \& Delgutte, 1996a, 1996b), a robust and pervasive correspondence was found between interspike interval statistics of populations of auditory nerve fibers and pitches that are produced by a wide array of complex tones. The auditory nerve is a strategic location for the study of pitch, the conduit through which must pass virtually all the auditory information that the central auditory system uses for the representation of sounds. Thus, whatever the nature of subsequent processing, the necessary information for all auditory capabilities must be present in the responses of auditory nerve fibers. For this reason, the auditory nerve has been one of the most intensively studied neural populations in the nervous system (Kiang et al., 1965; Rose et al., 1967).

\section{METHODS}

The auditory nerve responses presented here come from the same data set that has been published previously in (Cariani \& Delgutte, 1996a; 1996b), where experimental methods, stimuli, and analytical procedures are described in detail. Briefly, stimuli were numerically synthesized and delivered via closed, calibrated acoustic systems to Dialanesthetized cats with normal hearing. Posterior craniectomy and partial retraction of the cerebellum permitted the visually-guided insertion of glass microelectrodes into the auditory nerve near the internal auditory meatus. The auditory nerve in the cat consists of two populations of spiral ganglion afferents: myelinated Type I radial afferents ( $90 \%$ to 95\%) and unmyelinated Type II outer spiral afferents (5\% to 10\%) (Ryugo, 1992). The responses of single Type I auditory nerve fibers were recorded serially, using standard electrophysiological techniques. For each fiber, the characteristic frequency (CF), the discharge-rate response threshold, and the spontaneous rate were measured. The $\mathrm{CF}$ is the frequency to which a fiber has its lowest sound-pressure threshold (namely, the frequency for which the lowest sound-pressure level reliably elicits an extra spike within a $50 \mathrm{~ms}$ period).
Characteristic frequencies therefore provide an indication of the cochlear "place" from which an auditory nerve fiber receives its synaptic inputs. Units in the cochlear nucleus were recorded extracellularly, using tungsten electrodes positioned under direct visual guidance.

\section{NEURAL CORRELATES OF PITCH IN THE AUDITORY NERVE}

In these studies, microelectrode recordings were made of responses of single auditory nerve fibers to stimuli that produce low, periodicity pitches in humans. Figure 3 shows the responses of 51 auditory nerve fibers to 100 presentations of such a stimulus.

The waveform, power spectrum, and autocorrelation function of the vowel stimulus are shown in panels 3A,C,E. The vowel is a harmonic complex whose partials are all integer multiples of its fundamental frequency $(\mathrm{F} 0=80 \mathrm{~Hz})$ and whose waveform is periodic, repeating every fundamental period $(1 / \mathrm{F} 0=12.5) \mathrm{ms}$. Perceptually, the vowel produces a strong low pitch at its fundamental frequency $(\mathrm{F} 0=80 \mathrm{~Hz})$, whereas the vowel quality or timbre is determined by its single, formant frequency $(F 1=640 \mathrm{~Hz})$ and its bandwidth $(50 \mathrm{~Hz})$. The temporal patterns that are associated with the fundamental and the formant can be seen in the waveform (3A) of the vowel, whereas their respective harmonic spacings and concentration of energy in the formant can be seen in the power spectrum of the vowel (3C). The vowel stimulus was delivered at a moderate level (60 dB SPL).

Response peristimulus time histograms (PSTHs) for the whole ensemble of fibers are shown in Fig. 3B. The PSTHs are ordered by their respective characteristic frequencies (CFs). Immediately striking is the wide extent to which stimulus-driven temporal discharge patterns predominate over the entire auditory nerve array. Periodicities related to the fundamental F0, and hence, to the pitch period, are distributed across the entire array in the responses of fibers, with CFs ranging from $200 \mathrm{~Hz}$ to over $10 \mathrm{kHz}$. Given 

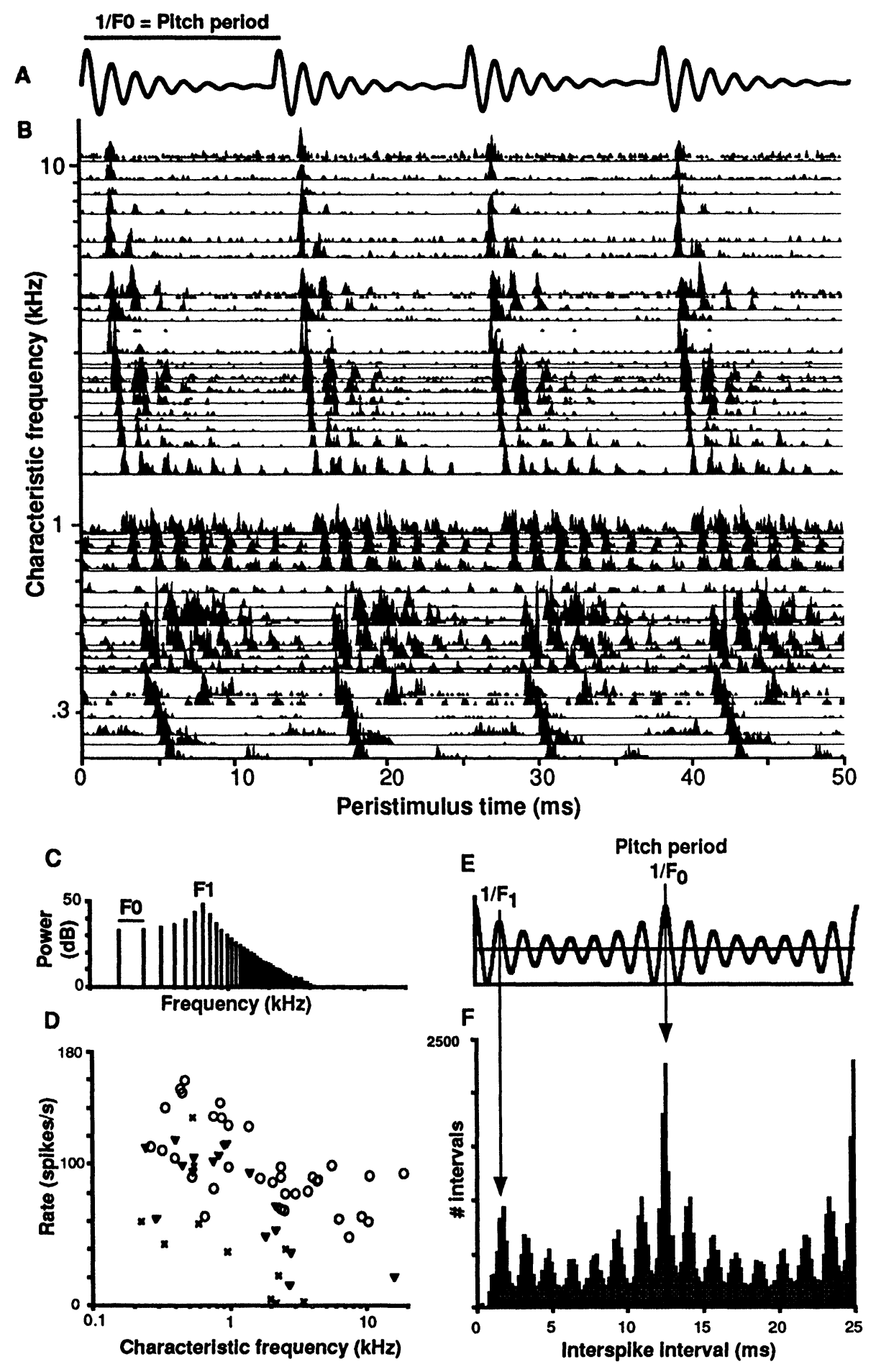

Fig. 3: Auditory nerve response to a single-formant vowel. A. Vowel waveform. A strong, low voice pitch is heard corresponding to the fundamental period, $1 / \mathrm{F} 0=12.5 \mathrm{~ms}$. B. Peristimulus time histograms of 51 cat auditory nerve fibers to 100 presentations at $60 \mathrm{~dB}$ SPL, arranged by characteristic frequency (CF). C. Power spectrum of the vowel (logarithmic frequency scale). The fundamental frequency, $(\mathrm{F} 0=80 \mathrm{~Hz}=$ frequency spacing of harmonics) and the formant frequency $(\mathrm{Fl}=640 \mathrm{~Hz}=$ spectral peak) are indicated. Bandwidth $=50 \mathrm{~Hz}$. D. Discharge rates as a function of CF and spontaneous rate (SR) (circles, high SR; triangles, medium SR; crosses, low SR). E. Stimulus autocorrelation function. Arrows indicate formant period 1/F1 and fundamental period 1/F0. F. Population-interval distribution formed by summing all-order intervals from all fibers. 
that the stimulus has relatively little power above $1 \mathrm{kHz}$ (Fig. 3C), this result is perhaps even more remarkable. To a greater or a lesser degree, all temporal discharge patterns follow the stimulus waveform, reflecting the relation between the respective fiber CFs and the stimulus spectrum. The reason for this near ubiquity of common temporal structure lies in the broadband nature of the responses at moderate- and high-stimulus levels and in the frequency asymmetry of cochlear tuning. The broad, low-frequency tails of tuning curves are such that low-frequency components presented at moderate levels ( $>50 \mathrm{~dB}$ SPL) can weakly drive large numbers of auditory nerve fibers whose CFs are well above them (Kiang \& Moxon, 1974; Kim \& Molnar, 1979). Discharge rates as a function of $\mathrm{CF}$ and spontaneous rate are shown in Fig. 3D for a rough comparison with the stimulus power spectrum. Spectral pattern representations for pitch that are based on discharge rates would require that

a) the individual harmonics be separated in population-rate profiles,

b) their frequencies be associated with the individual harmonics estimated, and

c) their harmonic relations be analyzed to infer the frequency of their common fundamental.

The two dominant periodicities of the vowel, $\mathrm{F} 0$ and $\mathrm{F} 1$, can be readily seen in the discharge patterns of fibers in different $C F$ regions. At this sound-pressure level, intervals related to the fundamental are found virtually everywhere, whereas formant-related periodicities are concentrated primarily in the CF regions that are nearest to the formant. More detailed views of the responses of two fibers with different CFs are shown in Fig. 4. A fiber whose $\mathrm{CF}$ is in the formant region $(\mathrm{CF}=950 \mathrm{~Hz}$, Fig. $4 \mathrm{~A}$ to $4 \mathrm{~F})$ discharges throughout most of each vowel period. A second fiber whose $\mathrm{CF}$ is above the formant region $(\mathrm{CF}=2100$ $\mathrm{Hz}$, Fig. $4 \mathrm{H}$ to $4 \mathrm{~K}$ ) responds less vigorously to the stimulus, producing spikes mostly at the onset of the vowel period. In Fig. 4. first-order and allorder interspike interval histograms are shown for the two fibers. A first-order interval histogram (Fig. 4E and 4J) tabulates the distribution of interspike intervals between consecutive spikes, whereas an all-order interval histogram ( $4 \mathrm{~F}$ and $4 \mathrm{~K}$ ), also called an autocorrelation histogram, tabulates the distribution of intervals between both consecutive and nonconsecutive spikes. Both fibers produce intervals that are related to the fundamental period $(1 / \mathrm{F} 0=12.5 \mathrm{~ms})$ and to components in the formant region $(1 / \mathrm{F} 1=1.6 \mathrm{~ms})$, albeit in different proportions.

It should be noted here that some measures that have been traditionally used to quantify temporal structure in neural responses, such as first-order interval distributions, period histograms, and synchronization indices, can provide misleading comparisons. For example, the discharge rate of the higher-CF fiber is more highly modulated, so that its period histogram would show spikes that are distributed over a smaller fraction of the vowel period, producing a correspondingly higher synchronization index. The higher-CF fiber might therefore be thought to better encode the fundamental period. Similarly, the higher-CF fiber produces more first-order intervals at the fundamental period than does its formant-region counterpart. But nevertheless, in absolute terms, the formant-region fiber contributes more all-order, F0-related interspike intervals to the population response. The reason for the inversions concerns the relative nature of these measures; for both measures, adding extra, intervening spikes alters the apparent amount of F0-related temporal structure. Because synchronization indices are relative, vectorial additions, adding extra spikes throughout the period, degrades the index. Because first-order interval distributions omit longer intervals when intervening spikes are present, these distributions systematically exclude longer F0-related intervals as discharge rates increase. As discharge rates generally increase with the level, if first-order intervals were used, then the neural representation of low fundamental frequencies should have worsened at higher levels, a trend that is not observed in the psychophysics. By contrast, allorder intervals that are associated with particular periodicities are not adversely affected by the extra, intervening spikes; hence such intervals 
A

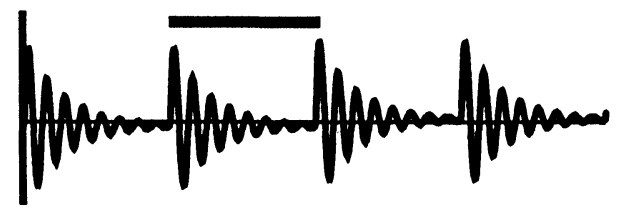

B

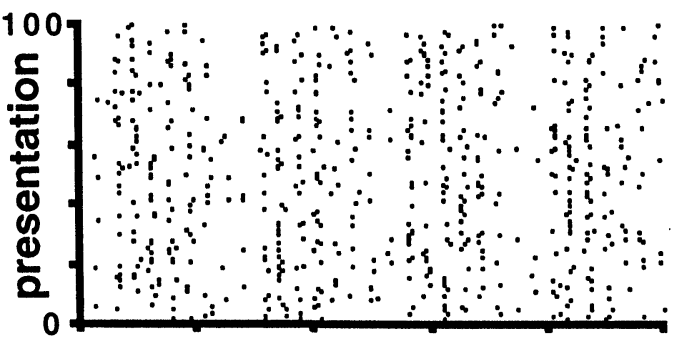

C

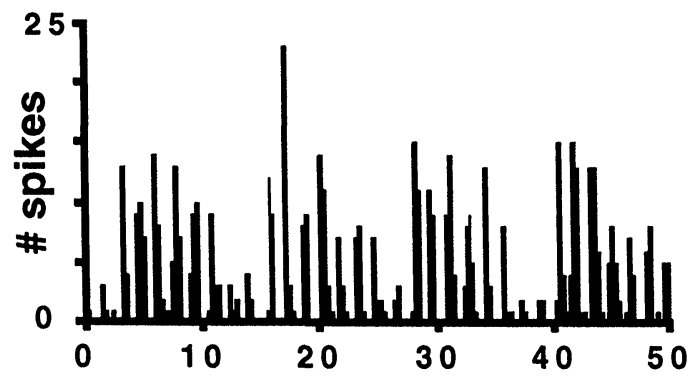

H

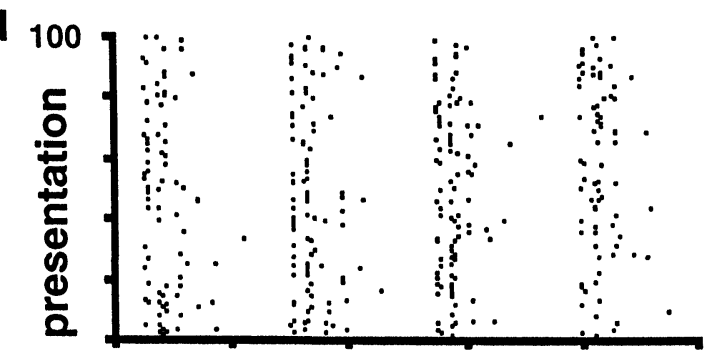

1

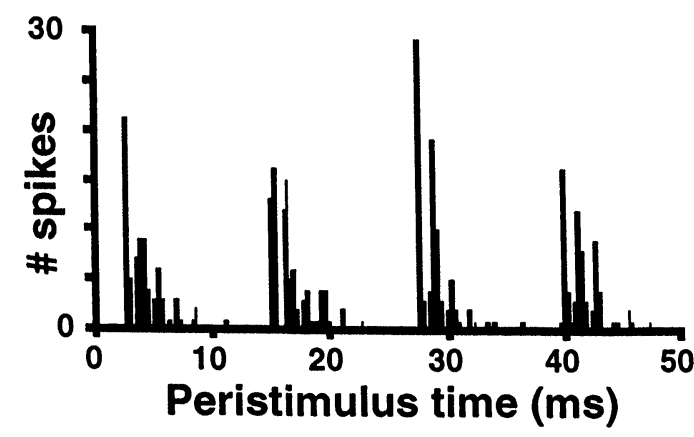

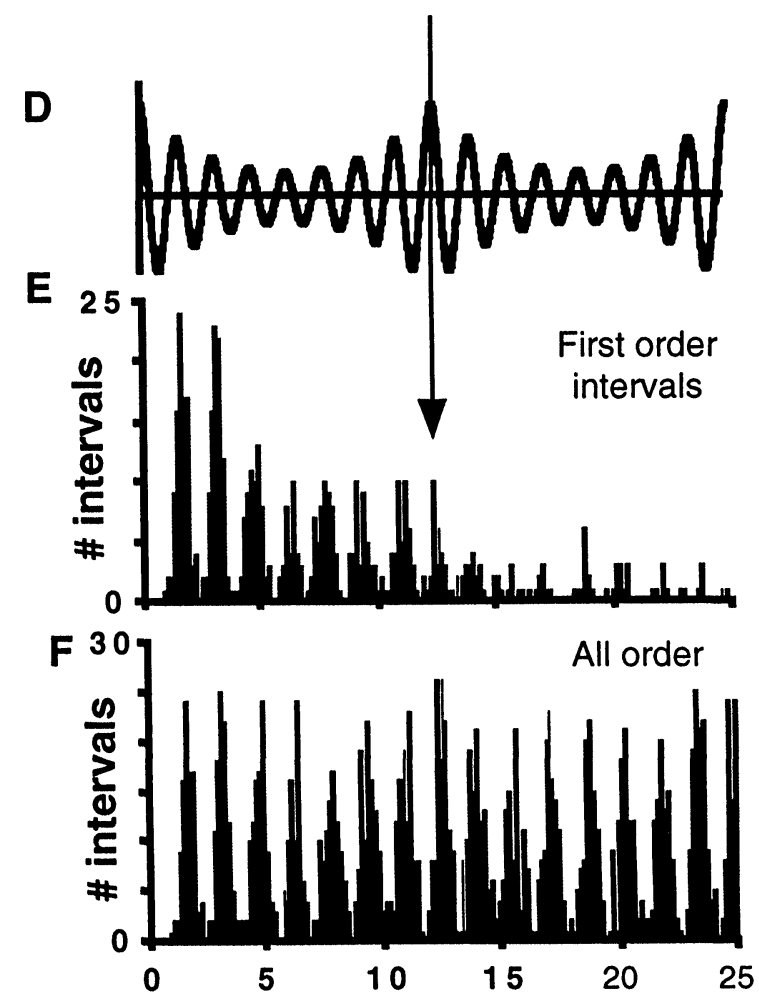
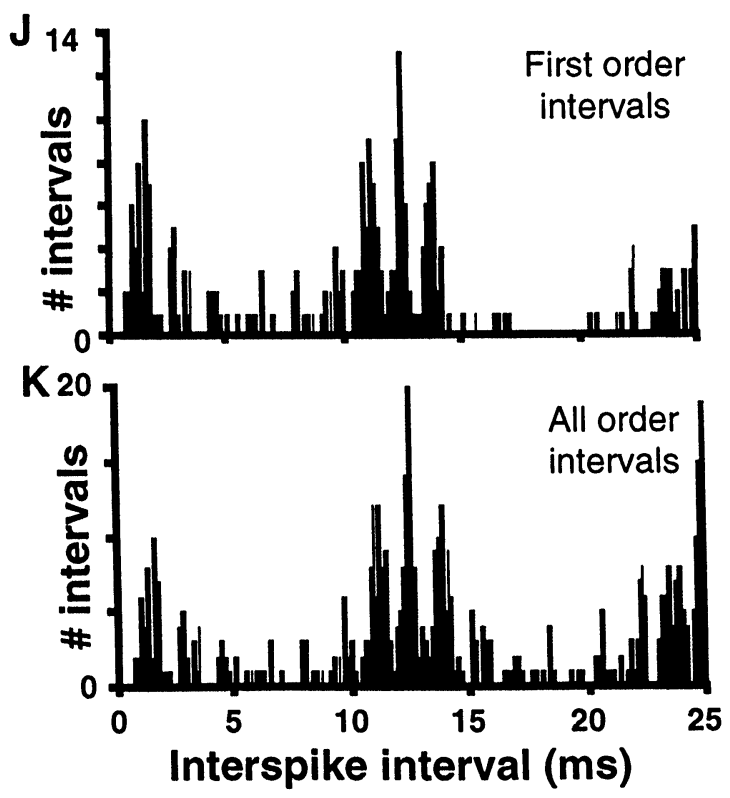

Fig. 4: Responses of two auditory nerve fibers with different CF's to 100 presentations of a single formant vowel, $60 \mathrm{~dB}$ SPL. A-F. Unit 25-19, CF $=950 \mathrm{~Hz}$, near the formant region. $\mathrm{H}-\mathrm{K}$. Unit $25-91, \mathrm{CF}=2.1 \mathrm{kHz}$, well above the formant region. A. Vowel waveform. Fundamental period 1/F0 (line) is $12.5 \mathrm{~ms}, \mathrm{~F} 0=80 \mathrm{~Hz}$. B. Dot raster display of individual spike arrival times. C. Peristimulus time histogram of spike arrival times. D. Stimulus autocorrelation function. Vertical line indicates fundamental period, $1 / \mathrm{F} 0=$ voice pitch. E. Histogram of first-order interspike intervals (between consecutive spikes). Arrows indicate intervals near the fundamental/pitch period. F. Histogram of all-order intervals (between both consecutive and nonconsecutive spikes). H-K. Corresponding histograms for the second fiber. 
constitute a neural coding strategy that better mimics perception in its behavior. For these reasons, it is important to choose measures of temporal response structure appropriate to the kinds of neural codes that one is investigating. Every neural response measure that one analyzes carries with it an implicit neural coding hypothesis.

Population-interval distributions are formed by summing together the all-order interspike interval distributions of individual fibers (Fig. $3 F$ ). Population-interval distribution serves as a rough estimate of the interval statistics of the entire auditory nerve. Because this distribution is the sum of many autocorrelation histograms or channel-auto-correlations such a distribution is often called the "summary autocorrelation" in many signal-processing and auditory simulations contexts (Meddis \& Hewitt, 1991; Lyon \& Shamma, 1995). The most salient aspect of this distribution is the large major peak associated with the fundamental period $(1 / \mathrm{F} 0=12.5 \mathrm{~ms})$ which, in turn, corresponds to the low pitch that is heard. For harmonic stimuli, all-order intervals at the fundamental period are always at least as numerous as those associated with any other periodicity (Rose, 1980), so that invariably, when the all-order intervals from many fibers are pooled together, the intervals at the fundamental are the most abundant. The second major peak, at $25 \mathrm{~ms}$, is also associated with the fundamental: the second peak corresponds to two fundamental periods. These major interval peaks correspond to the major peaks in the stimulus autocorrelation function (Fig. 3E). Thus, the most common interspike intervals that are generated at the level of the auditory nerve correspond to the pitch of the stimulus. This concordance was found to be the case for a wide range of fundamental frequencies and for many other kinds of harmonic stimuli as well, using both neurophysiological data (Cariani \& Delgutte, 1996a; 1996b) and auditory nerve simulations (Meddis \& Hewitt, 1991; Meddis \& O'Mard, 1997).

Yet another salient aspect of the populationinterval distribution (Fig. 3F) is its similarity in form with the autocorrelation function of the stimulus (Fig. 3E). The similar locations of major and minor peaks in both distributions is a general consequence of phase-locking, namely, temporal correlation between the stimulus waveform and the spike timings. In effect, population-interval distributions can serve as autocorrelation-like representations of the stimulus that contains the same information, up to the frequency limits of phase-locking, as its power spectrum. Thus, operations that are formally related to Fourier analysis can be neurally realized in the time domain by using all-order interval distributions.

Interspike interval information is extremely precise, permitting the fundamental period to be reliably estimated with a high degree of accuracy. From the responses of a few thousand spikes, estimates of the fundamental frequencies of stimuli producing strong pitches, such as the single-formant vowel, typically have standard errors on the order of $1 \%$. This estimate can be compared with the ability of human listeners ( $\sim 30,000$ Type I auditory nerve fibers) to distinguish fundamental frequencies differing by fractions of a percent (cf. Siebert, 1968; Siebert, 1970).

Many other aspects of pitch perception can be explained in terms of population-interval representations. Some of these are summarized in Fig. 5, with their associated population-interval histograms. Harmonic complexes lacking frequency components at their fundamentals, such as the AM tone in Fig. 5A, nevertheless evoke strong pitches at their "missing" fundamentals. The power spectrum of the AM tone in the second plot shows the frequencies of its three components (solid lines at 480, 640, and $800 \mathrm{~Hz}$ ) and the frequency of the low pitch heard at the fundamental (dotted line at $160 \mathrm{~Hz}$ ). Both the stimulus autocorrelations and the populationinterval distributions produced by such stimuli (rightmost plots) exhibit major peaks that correspond to these emergent pitches.

Different kinds of stimuli can give rise to the same low pitch. In one way or another, the auditory system creates strong perceptual equivalence classes for pitch. Population-interval distributions for four stimuli are shown in Fig. 5B. Despite very 
A Pitch of the
"missing
fundamental"
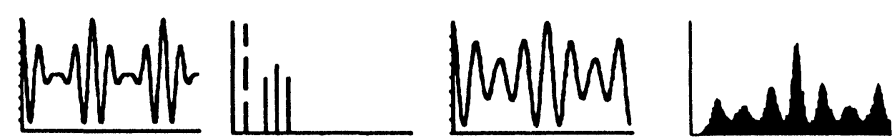

B Pitch Equivalence

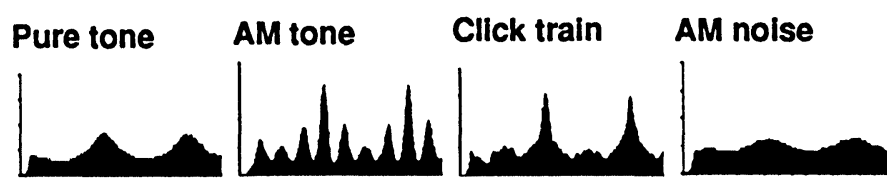

Strong pitches

C Pitch salience

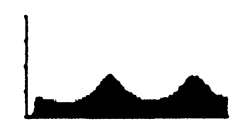

intivile
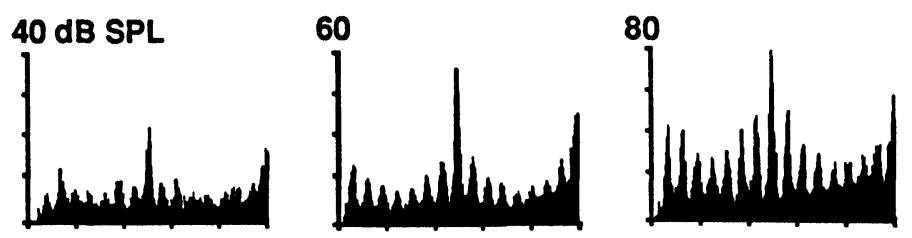

\section{E Pitch shift of inharmonic AM tones}

F Phase invariance

\section{$i_{1}^{n=6}$}
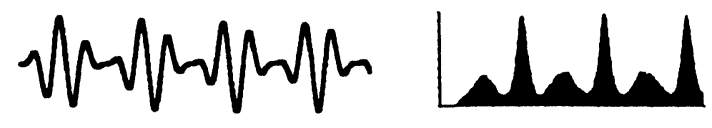

QFM tone

Weak pitch
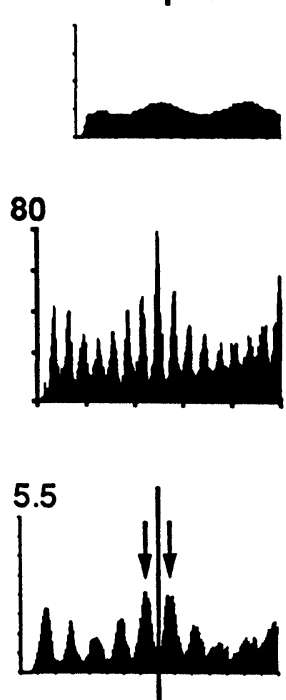

anlul
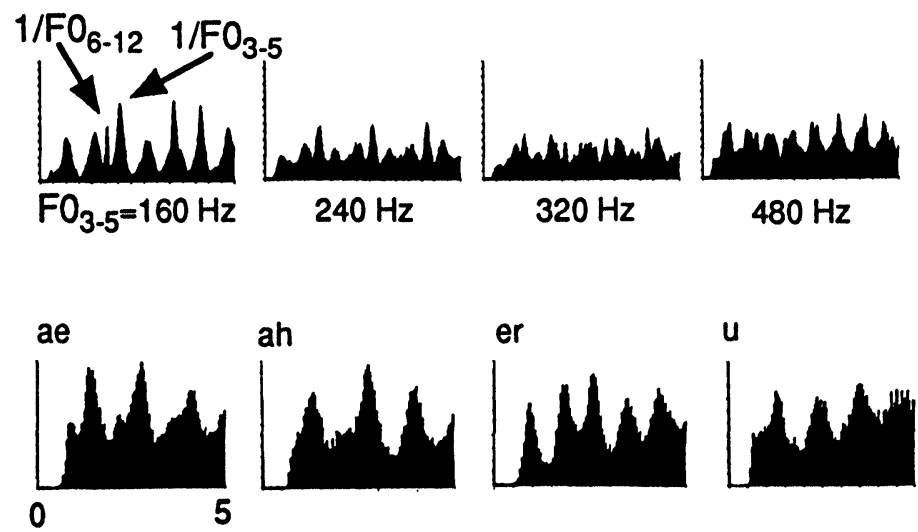

Fig. 5: Schematic summary of major correspondences between pitch percepts and population interval distributions at the level of the auditory nerve. The population-interval histograms plot relative numbers of all-order intervals (ordinates) of different durations (abcissas). Interval ranges for the histograms: 0-5 ms (H), 0-10 ms (A, F); 0-15 ms (B, C, E, G); 0-25 $\mathrm{ms}$ (D). Waveform segments are $20 \mathrm{~ms}$ long. See text for discussion. 
different power spectra, each kind of stimulus evokes a common low pitch at $160 \mathrm{~Hz}$. In all cases, the positions of the major interval peaks correspond to the common pitch period $(6.25 \mathrm{~ms})$. Thus, if the auditory system carried out an analysis of population-interval distributions, with the predominant interval corresponding to the pitch, then the pitch-equivalence of these stimuli would be a direct consequence of the basic neural-coding mechanisms that are used by the auditory system.

Different stimuli also differ in pitch salience, evoking stronger or weaker pitches. The population-interval distributions for three stimuli differing in pitch salience are shown in Fig. 5C. The two leftmost stimuli, a pure tone and an AM tone, evoked strong pitches, whereas the rightmost stimulus, an amplitude-modulated broadband noise, evoked a weak pitch. Qualitatively, the stimuli evoking strong pitches produced population-interval distributions with higher peak-to-mean ratios, namely, a higher fraction of all the pitch-related intervals that were produced. The stimuli producing weak pitches had low peak-to-mean ratios that were much closer to unity. The correspondence between pitch salience and peak-to-mean ratios is rough only because the pure tone produces a pitch that is always at least as salient as an equivalent AM tone, yet the peakto-mean ratio of the AM tone was substantially greater than that of the pure tone.

The low pitches of complex tones are highly invariant with respect to stimulus intensity. Population-interval distributions for the singleformant vowel discussed above are shown in Fig. 5D for three sound pressure levels: low (40 dB SPL), moderate (60 dB SPL) and high (80 dB SPL). Like human pitch judgments, the pitches that were estimated from population-interval distributions changed very little over the $40 \mathrm{~dB}$ range. Similarly, the representation of formantrelated periodicities remained very stable over that range. In the auditory system, such stability makes for extremely robust representations of pitch and timbre that do not degrade at moderate and high levels. In contrast, the saturation of discharge rates at these levels (Kim \& Molnar,
1979), with the consequent loss of representational contrast and precision, poses fundamental problems for rate-place coding of these qualities.

Population-based correlational representations of loudness are conceivable. As stimulus levels increase, population interval distributions more closely resemble the stimulus autocorrelation: the correlation coefficient $r$ between the stimulus autocorrelation function of the single formant vowel, and its respective population-interval histogram is 0.62 ( $\mathrm{n}=17$ fibers) for $40 \mathrm{~dB}$ SPL; rising to $0.70(n=61$ fibers) at $60 \mathrm{~dB}$ SPL, and $0.77(\mathrm{n}=31$ fibers) at $80 \mathrm{~dB}$ SPL. The correlation coefficient is, in effect, a measure of the amount of the common stimulus-driven time structure in the neural population. These comparisons are tentative because little overlap exists among the three sets of fibers. Nevertheless, such comparisons suggest a straightforward interpretation. As stimulus levels increase, a progressively greater fraction of discharge timing is stimulus-driven, such that the ratio of stimulus-driven intervals versus uncorrelated, spontaneously produced intervals steadily increases. Thus, the loudness of an auditory object potentially could be encoded by the fraction of the common temporally structured activity with which it is associated. Such a correlational representation would effectively use the entire dynamic range of the whole auditory nerve array. In such a scheme, spontaneous activity increases the dynamic range of the system by providing an uncorrelated noise source that can be successively displaced by stimulus-driven interspike intervals.

Complex pitch phenomena can also be explained in terms of population-interval distributions. Whereas periodic, harmonic tone complexes generally evoke unambiguous low pitches, inharmonic complexes can evoke ambiguous, multiple low pitches and small pitch shifts. A half-century ago, Schouten and deBoer (deBoer, 1976) conducted a classic set of experiments to determine whether pitch perception relies on spacings between adjacent frequency components (or equivalently on waveform envelope periods) rather than on harmonic relationships between components (or equivalently on the waveform fine structure). An AM tone consists of a complex comprising three successive harmonics that evokes a clear, 
unambiguous pitch at its (missing) fundamental frequency. When all three harmonics were shifted either upward or downward in frequency by the same amount, while keeping their frequency-spacings constant, the low pitch of the complex first shifted slightly by a much smaller amount than this frequency difference, an amount that was related to harmonic structure. When the frequencies were further shifted, listeners could hear one of two ambiguous pitches in the vicinity of the original pitch. The pitches estimated from the population-interval distributions for these respective cases (Fig. 5E) closely correspond to the pitch shifts that have been observed for human listeners. When the complex is harmonic $(\mathrm{n}=\mathrm{Fc} / \mathrm{Fm}=6=$ integer $)$, there is one clear pitch and one population-interval maximum. When the complex is inharmonic $(n=5.86=$ noninteger $)$, the pitch shifts, as does this maximum (arrow). When the components are further shifted downward $(n=5.5)$, either of the two pitches can be heard with roughly equal probability; correspondingly, two equal population-interval maxima are present (double arrows). Thus, a complex set of harmonically-based pitch effects can be readily explained in terms of population-interval representations.

The relative insensitivity of most auditory perception to the phase spectra of stationary sounds has long been recognized. For complex tones consisting of lower-frequency stimulus components $(<1500 \mathrm{~Hz})$, distinguishing stimuli that differ in phase, but not magnitude spectrum, despite very obvious differences in their waveforms, is generally very difficult. The waveforms of two such stimuli, an AM tone and a quasi-frequency modulated (QFM) tone that differ only in the phases of their center components $(640 \mathrm{~Hz})$, are shown in Fig. 5F. Their waveform envelopes are considerably different, with the AM tone having an envelope that is highly modulated, and the QFM tone having one that is much flatter. Their perceptual indistinguishability argues against auditory mechanisms that are sensitive to the phases of low-frequency components, such as neural computations that carry out an analysis of wholewaveform envelopes. The respective population- interval distributions for these stimuli are highly similar, almost indistinguishable.

Whereas the perception of pitches created by low-frequency harmonics is largely phaseinsensitive, the same cannot be said for the higher-frequency, closely-spaced, perceptually "unresolved" harmonics. Alterations in the phases of the upper harmonics can affect the low pitches that they evoke (for example, doubling the pitch) by altering the shapes of waveform envelopes that are produced by cochlear filtering. Thus psychophysically, two kinds of low pitches appear to be evoked by complex tones:

1) phase-insensitive pitches that are produced by lower-frequency, perceptually-resolved harmonics, and

2) phase-sensitive pitches that are produced by higher-frequency, unresolved harmonics.

This dichotomy has led some auditory theorists to posit dual pitch mechanisms, one for resolved harmonics alongside another for unresolved ones (Carlyon \& Shackleton, 1994; Shackleton \& Carlyon, 1994). Both sets of low pitches can be explained, however, in terms of a central analysis of all-order population-interval distributions. For closely spaced, unresolved, higher-frequency components, phase-locking to individual components is weak relative to phase-locking to envelopes, such that the interspike intervals that are produced (primarily by high CF fibers) mostly reflect the temporal structure of the envelope. The two kinds of low pitches may therefore correspond to the two modes by which pitchrelated interspike intervals can be generated: phase-locking to individual harmonics, and phase-locking to their interactions (envelopes). As population-interval distributions incorporate intervals that are generated by both mechanisms, these representations provide a unified analytical framework that accounts for both kinds of pitches (Cariani \& Delgutte, 1996a, 1996b; Meddis \& O'Mard, 1997). The perceptual resolvability of harmonics itself may have a neural basis in the two competing mechanisms of interval generation and in the discriminability of multiple interval peaks in population-interval distributions that they produce (cf. discussion of neural coding and 
signal detection (Moore, 1997, pp. 118-121). Thus, in population-interval accounts, linkages could exist between the perceptual resolvability of harmonics and different modes of pitch perception.

The dominance of lower-frequency harmonics in determining the low pitch of a complex tone ("the dominance region for pitch") can also be explained in population-interval terms. All other factors being equal, when two harmonic complexes, one consisting of lower-frequency $(<1500 \mathrm{~Hz})$ and the other of higher-frequency $(>1500 \mathrm{~Hz})$ components, each having slightly different fundamentals, are presented together so that their pitches compete, the pitch of the former is almost always heard over that of the latter. Population-interval distributions for such a combination of two complexes (harmonics 3-5 of fundamentals at $160,240,320$, and $480 \mathrm{~Hz}$ versus harmonics 6-12 of fundamentals $20 \%$ higher) are shown in (Fig. 5G). In all cases, the predominant interval in the distribution corresponds to the dominant pitch of the lower-frequency complex $\left(1 / \mathrm{F}_{3-5}\right)$ rather than that of the upper $\left(1 / \mathrm{F}_{6-12}\right)$. It thus appears that harmonics in the 500 to $1500 \mathrm{~Hz}$ range are disproportionately effective in generating many all-order interspike intervals at the fundamental period. These frequencies produce the most highly phase-locked responses in the greatest number of fibers. As a result, because of the basic factors that are common to many mammalian auditory systems, populationinterval distributions preferentially reflect the stimulus frequencies that play a predominant role in determining pitch percepts.

In addition to pitch, vowel quality or timbre can also be represented in population-interval distributions in patterns of short intervals (Fig. $5 \mathrm{H})$. Timbre itself is a complex, multidimensional auditory quality that can depend upon many factors, such as spectral shape, onset and offset properties, ongoing temporal dynamics (vibrato, roughness), and phase coherence (tones vs. noises). For stationary, harmonic sounds, timbre is determined by spectral shape, for example, the locations and heights of formants. The stimulus autocorrelation function and the population- interval distribution show a series of minor peaks, which are associated with components in the formant region that give the vowel its characteristic tone quality. Patterns of shorter intervals, those less than half the fundamental period $(1 / \mathrm{F} 0<6.25 \mathrm{~ms})$, reflect formant structure alone, whereas patterns of longer intervals reflect fundamental-formant relationships. For multiple formant vowels, the patterns of short intervals in population-interval distributions are sufficient to discriminate different vowels, using temporal information alone ( Palmer, 1992; Cariani, 1995; Cariani et al., 1997). The appearance and disappearance of minor peaks in the population-interval distribution also closely follow the perceptual vowelclass boundaries that are observed psychophysically (Hirahara et al., 1996).

These findings, when taken together with those derived from populations of simulated auditory nerve fibers, suggest that many diverse aspects of pitch perception can be directly explained in terms of population-interval distributions at the level of the auditory nerve. The main conclusions can be summarized as follows.

1) First, with very few exceptions, the most common all-order interval present in the population precisely and robustly corresponds to the pitch that is heard.

2) Second, the relative proportion of pitchrelated intervals amongst all others roughly corresponds to the strength of the pitch that is heard.

Many complex aspects of pitch perception can consequently be readily explained in terms of a central analysis of population-interval representations. All-order interspike intervals themselves are time durations that preserve harmonic relations between frequencies, such as the $2: 1$ octave ratio. If the auditory system uses representations that preserve the harmonic structure inherent in time intervals, then the perception of basic harmonic relations may be a direct consequence of the neural codes that the auditory system uses to represent and analyze sounds, rather than the product of elaborate harmonic cognitive schemas that have been built up from prior experience. 


\section{CODING OF PITCH IN THE CENTRAL AUDITORY SYSTEM}

Whether such a temporal analysis is in fact implemented in the central auditory system, what form it might take, and where it might occur are issues that are presently under investigation. Previous studies of neural responses in the auditory brainstem have indicated a widespread locking of discharges to pitch-related stimulus periodicities (Greenberg \& Rhode, 1987; Kim \& Leonard, 1988; Kim et al., 1990; Rhode, 1995). Several populations of neurons in the three major divisions of the cochlear nucleus (anteroventral division, AVCN; posteroventral division, PVCN; dorsal division, DCN) project to more central auditory stations in the brainstem and midbrain. By virtue of the differences in the distribution of their inputs and intrinsic properties, the neurons in each population have a characteristic response pattern when driven with pure tone bursts at their characteristic frequencies (TBCF). As in the auditory nerve, harmonic complex tones that produce strong pitches at their fundamentals similarly produce many pitch-related interspike intervals. Figure 6 shows the responses to a single-formant vowel of three physiologicallycharacterized units (Fig. 6A to 6C) that are representative of their respective populations.

Previous studies have identified the morphological cell-types that are associated with different TBCF response patterns (Pfeiffer, 1966; Rhode et al., 1983; Young, 1984). "Primary-like" TBCF responses are produced by spherical cells in the AVCN, sustained "chopper" responses to high-frequency tone bursts are produced by multipolar cells in the PVCN, whereas "pauser" patterns consisting of an onset-pause-sustained discharge pattern are produced by fusiform cells in the dorsal division (DCN). Primarylike units, as their name implies, have responses that are name implies, have responses that are most similar to those of primary sensory neurons (auditory nerve fibers). The discharges of this primarylike unit (Fig. 6A) exhibit stimulus-driven periodicities that are associated with fundamental $(12.5 \mathrm{~ms})$ and formant frequency (multiples of
$1.6 \mathrm{~ms}$ ), as well as with intrinsic periodicities that are associated with the characteristic frequency of the unit $(\mathrm{CF}=400 \mathrm{~Hz} ; 1 / \mathrm{CF}=2.5 \mathrm{~ms})$. These intrinsic periodicities ostensibly stem from similar CF-related periodicities that are seen in auditory nerve fibers, which are in turn produced by the mechanics of the cochlea. Sustained chopper responders are so named because they fire very regularly ("chop") at their own characteristic rate when driven by high-frequency tone bursts. When these units are driven by periodic harmonic stimuli, however, their discharges almost invariably lock strongly to the fundamental and only weakly to other stimulusperiodicities, if at all (Rhode, 1998). Pauser responders manifest more complex TBCF patterns that are the product of both intrinsic membrane properties and local circuit action. Whereas these units tend to respond more weakly. to periodic stimuli than do other cochlear nucleus response types, their discharges nevertheless lock to fundamentals to produce many pitch-related intervals. A general rule of thumb for these populations is that if a unit responds to a harmonic stimulus that is capable of producing a strong low pitch, the unit will either produce intervals that are related to the fundamental (extrinsic, stimulus-driven time structure) or to its characteristic frequency (stimulus-triggered, system-dependent intrinsic time structure). As intervals related to the fundamental are common to all units that are driven by a harmonic complex tone, but those related to any given characteristic frequency are not, it is all but inevitable that such pitch-related intervals predominate in these cochlear nucleus populations (for the same reasons that such intervals predominate in the auditory nerve). Thus, the population-interval representations of pitch appear to be viable at the level of the cochlear nucleus, as well as at the auditory nerve.

From all accounts, as one ascends the auditory pathway to auditory midbrain, thalamus, and cortex, the presence of pitch-related interspikeinterval information becomes less apparent. One possibility is that interspike interval information is converted to a rate-based representation 
somewhere in the pathway. Units that are differentially responsive to particular modulation frequencies have been proposed as the basis of such a time-to-place transformation (Langner, 1992), although whether such rate-based representations are sufficiently precise or robust to account adequately for pitch perception is not yet clear.

Another possibility is that interspike interval information persists, albeit in a sparser and more distributed form, at still more central stations. The same amount of interval information might well be distributed more sparsely over progressively greater numbers of neurons. Intervals bearing periodicity-related information might be multi- plexed with other kinds of spike patterns bearing information about location and context. These factors would make interspike interval information more difficult to detect using standard spike train analysis techniques.

Still another possibility is that central stations might simply use less interval information than is available at more peripheral stations. A great overabundance of interval-based information exists in the auditory nerve, such that relatively small numbers of intervals are sufficient to account for the high precision of frequency discrimination (Siebert, 1970). Indeed, this overabundance has often been used to assert that if auditory central processors were to make
A AVCN Primarylike
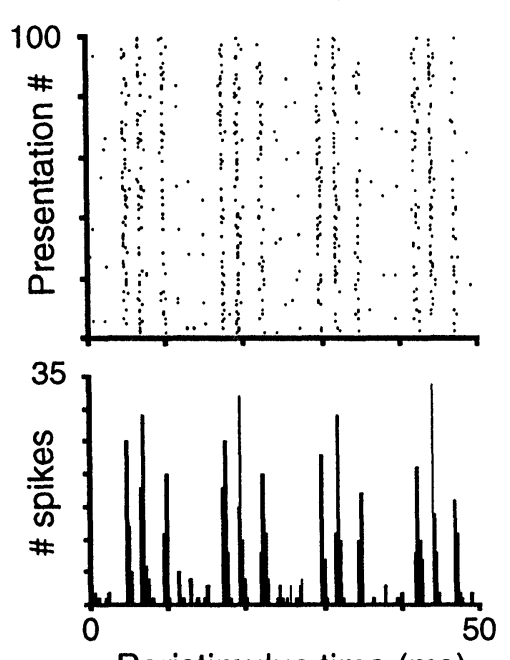

Peristimulus time (ms)

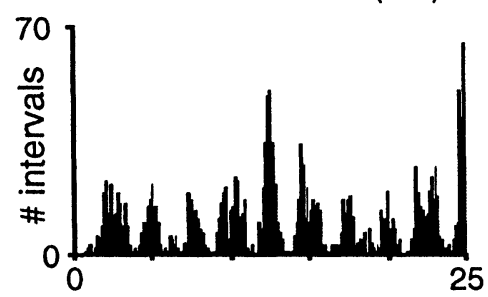

Interval (ms)
B PVCN Sustained chopper
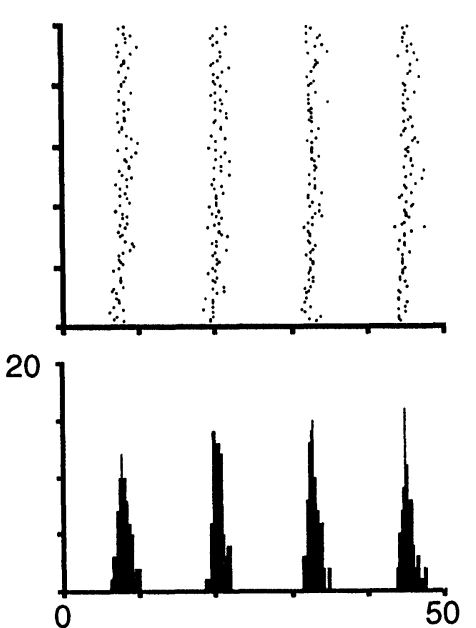

Peristimulus time (ms)

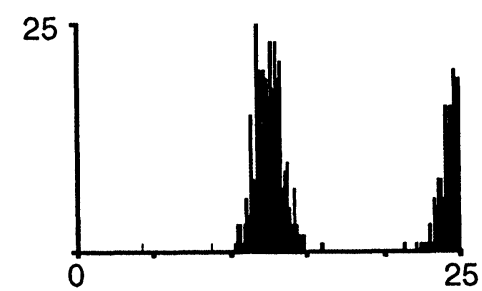

Interval (ms)
C DCN Pauser
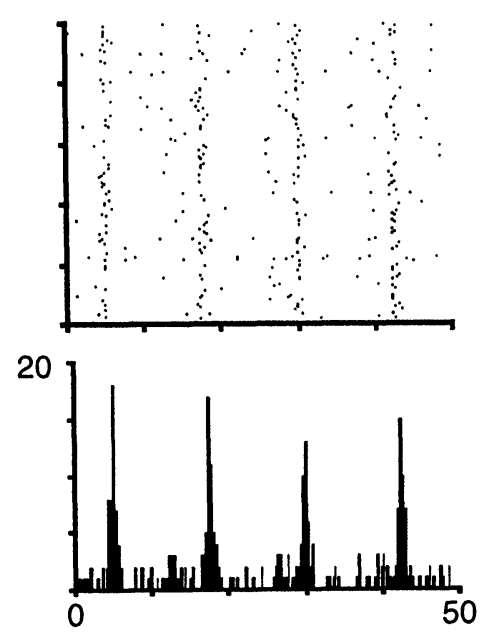

Peristimulus time (ms)

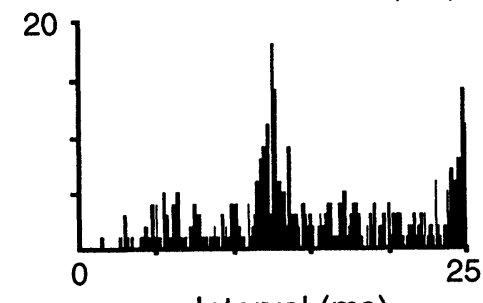

Interval (ms)

Fig. 6: Responses of three units in the cochlear nucleus to 100 presentations of a single-formant vowel $(\mathrm{F} 0=80 \mathrm{~Hz}, \mathrm{~F} 1=640 \mathrm{~Hz}$, $\mathrm{BW}=50)$ at $60 \mathrm{~dB}$ SPL. Units were classified according to their PSTH response to short tone bursts at CF. A. Dot-raster, PSTH, and all-order interval histogram for a primarylike unit in antero-ventral cochlear nucleus $(\mathrm{AVCN}), \mathrm{CF}=400 \mathrm{~Hz}$. B. Response of a sustained chopper unit in posterior-ventral cochlear nucleus (PVCN), $\mathrm{CF}=1.5 \mathrm{kHz}$. C. Response of a pauser unit in dorsal cochlear nucleus (DCN), $\mathrm{CF}=4.4 \mathrm{kHz}$. 
optimal use of this information, then human frequency discrimination would be some 40 times better than it is. The other side of this coin is that even if most interval information were to be lost or degraded in the ascending pathway, then enough information would remain to account for the observed precision of pitch discrimination.

Although stimulus-driven temporal structure declines at higher stations, it is important not to understate how much remains. Most studies thus far have been conducted under general anesthesia, but such agents generally reduce the upper frequency limits of stimulus-driven neural response periodicities by about one half (for example, see Goldstein et al., 1959). In unanesthetized animals, considerable phaselocking to $1-2 \mathrm{kHz}$ tones is observed at the thalamic level (de Ribaupierre, 1997). Likewise, in the input layers of unanesthetized primary auditory cortex, fundamental frequencies up to $400 \mathrm{~Hz}$ are reflected in the synchronized responses of local ensembles of auditory neurons (Steinschneider et al., 1998). First-spike latencies for onsets of tone bursts at the level of the primary auditory cortex have small variances on the order of fractions of a millisecond that are comparable to those seen at the auditory nerve (Phillips, 1989; Heil, 1997), despite a conspicuous lack of sustained phase-locking to the pure tones themselves. Precise temporal patterns, embedded in spike trains, occur in a diversity of cortical locations (Abeles et al., 1993; Lestienne \& Tuckwell, 1998). Although the evidence for and against time structure in the cerebral cortex has a decidedly mixed character, the data nevertheless suggest that the cortex may be capable of preserving more fine timing information than is commonly thought.

\section{GENERAL IMPLICATIONS FOR SENSORY CODING}

Population-interval representations hold many general implications for sensory coding. The coding of pitch through the interspike interval statistics of a population of neurons is a strong example of a temporal pattern code in its purest form, an example of a distributed temporal population code that does not entail interneural synchrony.

The population-interval distribution differs from both rate- and channel-based representations in two crucial ways: through the different nature of their primitives, and through the qualitatively different roles that channels play. Interspike intervals are time intervals that describe temporal relations between pairs of jointly occurring spike events. Such time intervals constitute correlational, relational primitives. In contrast, representations that are based on probabilities or on rates of unitary spike events count numbers of spike events over contiguous time windows. The counting assumption, with its scalar signals, in turn necessitates a whole host of assumptions concerning the functional topology of neural networks (Cariani, 1997). Second, in a population-interval representation, channel identities, which particular channels are activated how much, are not essential to the representational function. In the auditory nerve, of course, particular CF regions are preferentially activated by stimulus components that are nearby in frequency, and these regions will therefore contribute relatively more of their stimulusrelated intervals to the global distribution. In this way, the population-interval distribution reflects the differential contributions of different $\mathrm{CF}$ regions. Once the intervals are combined, however, the representation does not rely on the particular channel-identities of the fibers to encode frequency (because the intervals themselves bear this information, and in a much more precise and robust way). One could discard all information concerning characteristic frequency (or cochlear place) without affecting the representation. In contrast, in a channel-based neural representation, such as a rate-place frequency map, the identities of particular channels are absolutely critical for representational function. Consequently, stimulus representations would be corrupted if the channelidentities were scrambled (if the "labels" on the 
"labeled lines" were switched). Thus, the population-interval representation relies upon how neurons in a population respond and which intervals they produce, rather than upon which particular neurons fire how much.

The basic informational constituents of rateplace and population-interval representations are therefore very different, such that they complement each other, with neither representational mode precluding the other. The same holds true for representations based on relative response latencies and neural synchronies: fine temporal structure and relative latency patterns can all coexist within the coarser-grained, tonotopically ordered spatial patterns of activation. At the level of the auditory nerve and cochlear nucleus, the representation of periodicity pitch appears to follow this pattern of fine temporal structure within more coarsely tuned frequency channels.

Strong correspondences, population-interval distributions, and their respective stimulus autocorrelation functions were manifest in the similar patterns of major and minor peaks, with major peaks corresponding to pitch and minor peaks to timbres. Pitch judgments are relatively welldescribed by temporal autocorrelation models, precisely because the neural, interval-based representations subserving these judgments are themselves autocorrelation-like. In retrospect, the reasons for such similarities are fairly straightforward, being direct consequences of the stimulus-locked nature of auditory nerve fiber discharges. In the cochlea, the acoustic stimulus is, in effect, passed through a set of band-pass filters, such that each auditory nerve fiber is driven by a different set of frequency components. If a signal is passed through an array of overlapping frequency channels consisting of linear band-pass filters, then a series of filtered waveforms is produced. The sum of the channelautocorrelations of the filtered waveforms equals the autocorrelation of the original, unfiltered signal (Licklider, 1951) for the same formal reasons that permit the linear superposition of Fourier components in power spectra. In the cochlea, each auditory nerve fiber produces phase-locked discharges to components whose frequencies are closest to its own $\mathrm{CF}$, and in doing so, produces all-order intervals that are correlated with the autocorrelations of those components. The interval peak positions for individual fibers consequently mirror those in the stimulus autocorrelation (Fig. 4). In a linear system, both peak positions and relative heights would mirror those in the stimulus autocorrelation function. Whereas the populationinterval distributions presented in Fig. 3F show similar peak positions, the relative peak heights are noticeably different. Such differences are created by nonlinear processes, such as firing rate thresholds and saturations, that alter relative peak heights without changing peak positions. Representations of frequency that are based on all-order interspike intervals are therefore resistant to many kinds of intensity-dependent nonlinearities. The functional implications of nonlinear distortions in the cochlea thus depend critically on the neural codes that the central auditory system uses. The robustness of intervalbased representations, with respect to intensitydependent distortions, makes them ideal for representing auditory forms.

More generally, it can be said that to the extent that spike-arrival times are correlated with a stimulus waveform, the intervals between spikes will be correlated with the stimulus autocorrelation function. This relation will hold for any sensory system whose receptors follow the time courses of their effective stimuli. Such phase-locking is seen for patterns of vibrations on the skin (Morley et al., 1990; Mountcastle, 1993) and for changing luminance patterns as images move relative to retinal arrays ( Reichardt, 1961; Pollen et al., 1989; Bialek et al., 1991). Autocorrelation-like sensory representations that use all-order interspike intervals thus constitute potential stimulus-coding strategies in such modalities. Representations of visual form and texture that are based on spatial autocorrelation have been proposed (Uttal, 1975), but few attempts have been made to use stimulus-driven, fine spatiotemporal correlation structure for this purpose (Reitboeck et al., 1988). Recent psychophysical evidence points to a strong role for such 
structure in the perception of visual forms (Lee \& Blake, 1999).

The means by which neural computational architectures might make use of interspike interval statistics of populations of neurons largely remains to be explored. Different kinds of codes naturally lead to different neural-processing architectures. Channel-coding naturally leads to connectionist networks, in which information is represented through specific patterns of channel activation and processed through networks, in which specific connectivities determine functional roles. In such systems, highly specific modification of effective connectivity is the main mechanism by which functional plasticity is achieved.

For the most part, when temporal structure has been considered in functional terms; it has been assumed that to use the information, temporal patterns must be converted to channel-activation patterns. Thus the first neural auditory computation networks converted time-of-arrival differences and temporal patterns into spatial patterns of activations. Time-delay neural architectures, consisting of tapped delay lines and coincidence counters, were proposed for using interaural time-of-arrival differences to localize sounds by computing binaural cross-correlations (Jeffress, 1948). The coincidence channels that were maximally activated served to indicate the relative time-of-arrival of sounds at the two ears and hence, their location in the azimuthal plane. Similarly, neural time-delay networks that used a different arrangement of tapped delay lines and coincidence counters were proposed for carrying out neural autocorrelational analyses that spatialize population-interval distributions for analysis (Licklider, 1951; Lyon \& Shamma, 1995). The coincidence counters act as autocorrelating periodicity-detectors that operate on all-order intervals.

In general, systematic differences in arrival times of external disturbances at different sensory surfaces support neural representations for external location based on stimulus-locked, timeof-arrival codes. Such differences naturally lend themselves to analysis via temporal crosscorrelation operations (Carr, 1993). On the other hand, characteristic temporal spike-patterns that are generated at sensory surfaces through either stimulus-locking or stimulus-triggered intrinsic responses potentially support neural representations of stimulus-form. These characteristic temporal patterns naturally lend themselves to autocorrelational analyses. Early comprehensive computational models for hearing (Licklider, 1959; Cherry, 1961) integrated both kinds of correlational processes to represent both location and form. How many auditory functions can be subsumed under these two operations, and the extent to which other sensory systems might operate using similar principles, remains to be seen.

In time-delay architectures, plasticity of function is achieved by adjusting effective connectivities to favor particular sets of timedelays (Licklider, 1959; Tank \& Hopfield, 1987), or by adjusting time delays to synchronize particular sets of inputs (MacKay, 1962). Changes in temporal response properties as a result of conditioning have been observed in a wide variety of systems (Morrell, 1967; Thatcher \& John, 1977; John \& Schwartz, 1978; Singer, 1995). In principle, a neural assembly can be formed that will respond preferentially to any spatiotemporal pattern in its inputs by adjusting the relative time delays and connection weights to match those in the incoming pattern. Neural delays can be created by any process that takes time to unfold and be modified by any process that alters response latency. Axonal and dendritic transmission times, latencies of activation, and timecourses of neuronal recovery (Raymond, 1979; Wasserman, 1992) potentially provide shifts in time and sensitivities to time pattern that can become control points for adaptive adjustment. Intraneural delays can then be concatenated in multisynaptic, recurrent, and/or re-entrant pathways to form still longer delays. To the extent that timing is important in a neural information processing system, such alterations of temporal response properties provide avenues by which modifications of structure can lead to modifications of function.

Finally, neural networks that carry out their operations entirely within the time domain can be 
envisioned (Cariani, in press). Neural timing nets, consisting of tapped delay lines and coincidence detectors, analyze temporally-coded inputs to produce temporally-coded outputs. Simple feedforward timing networks function as temporal sieves that extract common periodicities in their inputs, thereby finding similarities and differences between them. A fundamental advantage of these timing nets is that they operate on interval statistics, obviating the necessity for precise regulation of point-to-point connectivities. Recurrent timing networks can be used to build up periodic temporal patterns in their inputs and to separate out repeating patterns that have different periods. Combinations of feed-forward and recurrent delay lines coupled with coincidence and anticoincidence elements may then provide general-purpose strategies for detecting correlational, relational structure in the world. Efforts to understand the combined functional capabilities of temporal codes and timing nets are presently in their early, formative stages.

\section{ACKNOWLEDGMENTS}

This work was supported by Grant DC03054 from the National Institute for Deafness and Communications Disorders (NIDCD) of the National Institutes of Health $(\mathrm{NIH})$.

\section{REFERENCES}

Abeles M, Bergman H, Margalit E, Vaadia E. Spatiotemporal firing patterns in the frontal cortex of behaving monkeys. J. Neurophysiol. 1993; 70: 1629-1638.

Barlow HB. The neuron doctrine in perception. In: Gazzaniga, MS, ed, The Cognitive Neurosciences. Cambridge: MIT Press 1995; 415-435.

Bialek W, Rieke F, van Stevenink RR, de Ruyter WD. Reading a neural code. Science 1991; 252: 1854-1856.

Boring EG. Sensation and Perception in the History of Experimental Psychology. New York: AppletonCentury-Crofts, 1942.

Bower TGR. The evolution of sensory systems. In: MacLeod RB, Pick Jr. H, eds, Perception: Essays in
Honor of James J. Gibson. Ithaca, NY: Cornell University Press 1974; 141-152.

Brugge JF, Reale RA, Hind JE. The structure of spatial receptive fields of neurons in primary auditory cortex of the cat. J Neurosci 1996; 16: 4420-4437.

Cariani P. As if time really mattered: temporal strategies for neural coding of sensory information. Communication and Cognition-Artificial Intelligence (CC-AI) 1995; 12: 161-229. Reprinted in: Pribram K, ed, Origins: Brain and Self-Organization. Hillsdale, NJ: Lawrence Erlbaum 1994; 208-252.

Cariani P. Emergence of new signal-primitives in neural networks. Intellectica 1997; 1997: 95-143.

Cariani P. Temporal coding of sensory information. In: Bower, JM, ed, Computational Neuroscience: Trends in Research. 1997. New York: Plenum 1997; 591-598.

Cariani P. Neural timing nets for auditory computation. In: Greenberg S, Slaney M, eds, Computational Models of Auditory Function. Amsterdam: IOS Press in press; 16 pp.

Cariani P. Delgutte B. Tramo M. Neural representation of pitch through autocorrelation. Proceedings. Audio Engineering Society Meeting (AES). New York. September. 1997. Preprint \#4583 (L-3); 1997.

Cariani PA, Delgutte B. Neural correlates of the pitch of complex tones. I. Pitch and pitch salience. J Neurophysiol. 1996a; 76: 1698-1716.

Cariani PA, Delgutte B. Neural correlates of the pitch of complex tones. II. Pitch shift. pitch ambiguity. phaseinvariance. pitch circularity. and the dominance region for pitch. J Neurophysiol. 1996b; 76: 1717-1734.

Carlyon RP. Shackleton TM. Comparing the fundamental frequencies of resolved and unresolved harmonics: evidence for two pitch mechanisms?. J Acoust. Soc. Am. 1994; 95: 3541-3554.

Carr CE. Processing of temporal information in the brain. Ann Rev Neurosci 1993; 16: 223-243.

Cherry C. Two ears- but one world. In: Rosenblith WA, ed, Sensory Communication. New York: MIT Press/ John Wiley 1961; 99-117.

Chung SH. Raymond SA. Lettvin JY. Multiple meaning in single visual units. Brain Behav Evol 1970; 3: 72-101.

Covey E. Temporal Neural Coding in Gustation. Duke University. 1980.

de Boer E. On the "residue" and auditory pitch perception. In: Keidel WD, Neff WD, eds, Hand-book of Sensory Physiology. Berlin: Springer Verlag 1976; 479-583.

de Cheveigné A. A pitch perception model. ICASSP 86 (Tokyo) 1986; 897-900.

de Ribaupierre F. Acoustical information processing in the auditory thalamus and cerebral cortex. In: Ehret G, Romand R, eds, The Central Auditory System. New York: Oxford University Press 1997; 317-397.

Deadyler SA, Hampson RE. Ensemble activity and behavior: what's the code?. Science 1995; 270: 1316-1318. 
Delgutte B. Physiological models for basic auditory percepts. In: Hawkins $\mathrm{H}$, McMullin T, Popper AN, Fay RR, eds, Auditory Computation. New York: Springer Verlag, 1995, 157-220.

Di Lorenzo PM, Hecht GS. Perceptual consequences of electrical stimulation in the gustatory system. Behavioral Neuroscience 1993; 107: 130-138.

Emmers R. Pain: A Spike-Interval Coded Message in the Brain. New York: Raven Press 1981.

Evans EF. Place and time coding of frequency in the peripheral auditory system: some physiological pros and cons. Audiology 1978; 17: 369-420.

Gerstner W. Spiking neurons. In: Maass W, Bishop CM, eds, Pulsed Neural Networks. Cambridge, Massachusetts, USA: MIT Press 1999; xiii-xxvi.

Gesteland RC, Lettvin JY, Pitts WH. Chemical transmission in the nose of the frog. J Physiol. 1965; 181: 525-559.

Goldstein MH Jr, Kiang NYS, Brown RM. Responses of the auditory cortex to repetitive acoustic stimuli. J Acoust Soc Am 1959; 31: 356-364.

Greenberg S, Rhode WS. Periodicity coding in cochlear nerve and ventral cochlear nucleus. In: Yost WA, Watson CS, eds, Auditory Processing of Complex Sounds. Hillsdale, NJ: Lawrence Erlbaum Associates $1987 ; 225-236$.

Hatsopoulos NG, Ojakangas CL, Donohue JP, Maynard EM. Detection and identification of ensemble codes in motor cortex. In: Eichenbaum HB, Davis JL, eds, Neuronal Ensembles: Strategies for Recording and Decoding. New York: Wiley-Liss 1998; 161-176.

Heil P. First-spike timing of auditory-nerve fibers and comparison with auditory cortex. J Neurophysiol. 1997; 78: 2438-2454.

Hirahara T, Cariani P, Delgutte B. Representation of lowfrequency vowel formants in the auditory nerve. Proceedings. European Speech Communication Association (ESCA) Research Workshop on The Auditory Basis of Speech Perception. Keele University, UK, July 1519, 1996; 1-4.

Jeffress LA. A place theory of sound localization. J Comp Physiol Psychol 1948; 41: 35-39.

John ER. Switchboard vs. statistical theories of learning and memory. Science 1972; 177: 850-864.

John ER. Representation of information in the brain. In: John ER, ed, Machinery of the Mind. Boston, MA: Birkhauser 1990; 27-56.

John ER, Schwartz EL. The neurophysiology of information processing and cognition. Ann Rev Psychol 1978; 29: 1-29.

Kauer JS. Response patterns of amphibian olfactory bulb neurones to odour stimulation. J Physiol 1974; 243: 695-715.

Kiang NYS. Stimulus representation in the discharge patterns of auditory neurons. In: Tower DB, ed, The Nervous System. New York: Raven Press 1975; 81-96.
Kiang NYS. Moxon EC. Tails of tuning curves of auditorynerve fibers. J Acoust Soc Am 1974; 55: 620-630.

Kiang NYS, Watanabe T, Thomas EC. Clark LF. Discharge Patterns of Single Fibers in the Cat's Auditory Nerve. Cambridge, Massachusetts, USA: MIT Press 1965.

Kim DO, Leonard G. Pitch-period following response of cat cochlear nucleus neurons to speech sounds. In: Duifhuis $\mathrm{H}$, Horst JW, Wit HP, eds, Basic Issues in Hearing. London: Academic Press 1988; 252-260.

Kim DO, Molnar CE. A population study of cochlear nerve fibers: comparison of spatial distributions of average-rate and phase-locking measures of responses to single tones. J Neurophysiol. 1979; 42: 16-30.

Kim DO, Sirianni JG, Chang SO. Responses of DCN-PVCN neurons and auditory nerve fibers in unanesthetized decerebrate cats to AM and pure tones: Analysis with autocorrelation/power-spectrum. Hearing Res 1990; 45: 95-113.

Kozak WM, Reitboeck HJ. Color-dependent distribution of spikes in single optic tract fibers of the cat. Vision Res 1974; 14: 405-419.

Langner G. Periodicity coding in the auditory system. Hearing Res 1992; 60: 115-142.

Laurent G, Naraghi M. Odorant-induced oscillations in mushroom bodies of the locust. J Neurosci. 1994; 14 : 2993-3004.

Lee D, Port NL, Kruse W, Georgoupoulos AP. Neuronal population coding: multi-electrode recordings in primate cerebral cortex. In: Eichenbaum HB, Davis J, eds, Neuronal Ensembles: Strategies for Re-cording and Decoding. New York: Wiley-Liss 1998; 117-138.

Lee S-H, Blake R. Visual form created solely from temporal structure. Science 1999; 284: 1165-1168.

Lestienne R, Strehler BL. Time structure and stimulus dependence of precise replicating patterns present in monkey cortical neuronal spike trains. Brain Res 1987; 43: 214-238.

Lestienne R, Tuckwell HC. The significance of precisely replicating patterns in mammalian CNS spike trains. Neuroscience 1998; 82: 315-336.

Licklider JCR. A duplex theory of pitch perception. Experientia 1951; VII: 128-134.

Licklider JCR. Three auditory theories. In: Koch S, ed, Psychology: A Study of a Science. Study I. Conceptual and Systematic. New York: McGraw-Hill 1959; 41-144.

Lyon R, Shamma S. Auditory representations of timbre and pitch. In: Hawkins H, McMullin T, Popper AN, Fay RR, eds, Auditory Computation. New York: Springer Verlag 1995; 221-270.

MacKay DM. Self-organization in the time domain. In: Yovitts, MC, Jacobi GT, Goldstein GD., eds, SelfOrganizing Systems 1962. Washington. DC: Spartan Books 1962; 37-48.

Macrides F. Dynamic aspects of central olfactory 
processing. In: Schwartze DM, Mozell MM, eds, Chemical Signals in Vertebrates. New York: Plenum 1977; 207-229.

Macrides F, Chorover SL. Olfactory bulb units: activity correlated with inhalation cycles and odor quality. Science 1972; 175: 84-86.

Marion-Poll F, Tobin TR. Temporal coding of pheromone pulses and trains in Manduca sexta. J Comp Physiol A 1992; 171: 505-512.

Meddis R. Hewitt MJ Virtual pitch and phase sensitivity of a computer model of the auditory periphery. I. Pitch identification. J Acoust Soc Am 1991; 89: 2866-2882.

Meddis R, O'Mard L. A unitary model of pitch perception. J Acoust. Soc. Am. 1997; 102: 1811-1820.

Moore BCJ. Introduction to the Psychology of Hearing, 4th Ed. London: Academic Press 1997; 118-121.

Morley JW, Archer JS, Ferrington DG, Rowe MJ, Turman AB. Neural coding of complex tactile vibration. Information Processing in Mammalian Auditory and Tactile Systems. New York: Alan R. Liss 1990; 127-140.

Morrell F. Electrical signs of sensory coding. In: Quarton GC, Melnechuck T, Schmitt FO, eds, The Neurosciences: A Study Program. New York: Rockefeller University Press 1967; 452-469.

Mountcastle V. The problem of sensing and the neural coding of sensory events. In: Quarton GC, Melnechuk T, Schmitt FO, eds, The Neurosciences: A Study Program. New York: Rockefeller University Press 1967; 393-497.

Mountcastle V. Temporal order determinants in a somatosthetic frequency discrimination: sequential order coding. Annals New York Acad Sci 1993; 682: 151-170.

Onoda N, Mori K. Depth distribution of temporal firing patterns in olfactory bulb related to air intake cycles. $\mathrm{J}$ Neurophysiol 1980; 44: 29-39.

Palmer AR. Segregation of the responses to paired vowels in the auditory nerve of the guinea pig using autocorrelation. In: Schouten MEH, ed, The Auditory Processing of Speech. Berlin: Mouton de Gruyter 1992; 115-124.

Perkell DH, Bullock TH. Neural Coding. Neurosciences Research Program Bulletin 1968; 6: 221-348.

Pfeiffer RR. Classification of response patterns of spike discharges from units in the cochlear nucleus: tone-burst stimulation. Exp Brain Res 1966; 1: 220-235.

Phillips DP. Timing of spike discharges in cat auditory cortex neurons: implications for encoding of stimulus periodicity. Hearing Res 1989; 40: 137-146.

Pollen DA, Gaska JP, Jacobson LD. Physiological constraints on models of visual cortical function. In: Cotterill RMJ, ed, Models of Brain Function. Cambridge, UK: Cambridge University Press 1989; 115-136.

Raymond SA. Effects of nerve impulses on threshold of frog sciatic nerve fibres. J Physiol (Lond) 1979; 290: 273-303.

Reichardt W. Autocorrelation. a principle for the evaluation of sensory information by the central nervous system. In: Rosenblith WA, ed, Sensory Communication. New
York: MIT Press/John Wiley 1961; 303-317.

Reitboeck HJ Pabst M. Eckhorn R. Texture description in the time domain. In: Cotterill RMJ, ed, Computer Simulation in Brain Science. Cambridge, UK, Cambridge University Press 1988.

Rhode WS. Interspike intervals as correlates of periodicity pitch in cat cochlear nucleus. J Acoust Soc Am 1995; 97 : 2414-2429.

Rhode WS. Neural encoding of single-formant stimuli in ventral cochlear nucleus of the chinchilla. Hearing Res 1998; 117: 39-56.

Rhode WS, Smith PH, Oertel D. Physiological response properties of cells labeled intracellularly with horseradish peroxidase in cat ventral cochlear nucleus. J Comp Neurol 1983; 213: 448-463.

Richmond BJ, Gawne TJ. The relationship between neuronal codes and cortical organization. In: Eichenbaum HB, Davis JL, eds, Neuronal Ensembles: Strategies for Recording and Decoding. New York: Wiley-Liss 1998; 57-80.

Richmond BJ, Optican LM, Podell M, Spitzer H. Temporal encoding of two-dimensional patterns by single units in primate inferior temporal cortex. I. Response characteristics.. J Neurophysiol 1987; 57: 132-146.

Rieke F, Warland D, de Ruyter van Steveninck R, Bialek W. Spikes: Exploring the Neural Code. Cambridge. MA, USA: MIT Press 1997; 395.

Rose JE. Neural correlates of some psychoacoustical experiences. In: McFadden D, ed, Neural Mechanisms of Behavior. New York: Springer Verlag 1980; 1-33.

Rose JE, Brugge JR, Anderson DJ, Hind JE. Phase-locked response to low-frequency tones in single auditory nerve fibers of the squirrel monkey. J Neurophysiol 1967; 30: 769-793.

Ryugo DK. The auditory nerve: peripheral innervation. cell body morphology. and central projections. In: Webster DB, Popper AN, Fay RR, eds, The Mammalian Auditory Pathway: Neuroanatomy. New York: Springer-Verlag 1992; 23-65.

Shackleton TM, Carlyon RP. The role of resolved and unresolved harmonics in pitch perception and frequency modulation discrimination. J Acoust Soc Am 1994; 95: 3529-3540.

Shadlen MN, Newsome WT. Noise. neural codes and cortical organization. Curr Op Neurobiol 1994; 4: 569-579.

Shadlen MN, Newsome WT. The variable discharge of cortical neurons: implications for connectivity. Computation. and information coding. J Neurosci 1998; 18: 3870-3896.

Siebert WM. Stimulus transformations in the peripheral auditory system. In: Kollers PA, Eden M, eds, Recognizing Patterns. Cambridge, Massachusetts, USA: MIT Press 1968; 104-133.

Siebert WM. Frequency discrimination in the auditory system: place or periodicity mechanisms? Proc IEEE 1970; 58: 723-730. 
Singer W. Search for coherence: a basic principle of cortical self-organization. Concepts Neurosci 1990; 1: 1-26.

Singer W. Development and plasticity of cortical processing architectures. Science 1995; 270: 758-764.

Singer W. Putative functions of temporal correlations in neocortical processing. In: Koch C, Davis CL, eds, Large-Scale Neuronal Theories of the Brain. Cambridge, MA, USA: MIT Press 1995; 201-237.

Slaney M, Lyon RF. On the importance of time-a temporal representation of sound. In: Cooke M, Beet S, Crawford M, eds, Visual Representations of Speech Signals. New York: Wiley 1993; 95-118.

Srulovicz P, Goldstein JL. Central spectral patterns in aural signal analysis based on cochlear neural timing and frequency filtering. IEEE, Tel Aviv. Israel, 1977.

Steinschneider M. Reser DH. Fishman YI. Arezzo J. Click train encoding in primary auditory cortex of the awake monkey: Evidence for two mechanisms subserving pitch perception. J Acoust Soc Am 1998; 104: 2395-2955.

Stevens SS. Sensory power functions and neural events. In: Loewenstein WR, ed, Principles of Receptor Physiology. Berlin: Springer-Verlag 1971; 226-242.

Tank DW, Hopfield JJ Neural computation by concentrating information in time. Proc Natl Acad Sci USA 1987; 84 1896-1900.

Thatcher RW. John ER. Functional Neuroscience. Vol. I. Foundations of Cognitive Processes. Hillsdale, New Jersey, USA: Lawrence Erlbaum 1977; 382.

Troland LT. The psychophysiology of auditory qualities and attributes. 1929; 2: 28-58.

Uttal WR. The Psychobiology of Sensory Coding. New York: Harper Row 1973.

Uttal WR. An Autocorrelation Theory of Form Detection. New York: Wiley 1975.

van Noorden L. Two channel pitch perception. In: Clynes M, ed, Music. Mind and Brain. New York: Plenum 1982; 251-269.

Wasserman GS. Isomorphism. task dependence. and the multiple meaning theory of neural coding. Biol Signals 1992; 1: 117-142.

Wever EG. Theory of Hearing. New York: Wiley 1949.

Young ED. Response characteristics of neurons in the cochlear nuclei. In: Berlin C, ed, Hearing Science. San Diego, CA, USA: College Hill 1984; 423-460.

Young RA. Some observations on temporal coding of color vision: psychophysical results. Vision Res 1977; 17: 957. 

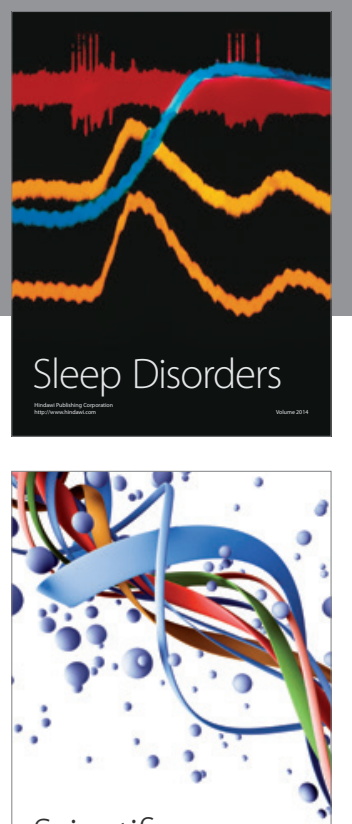

Scientifica
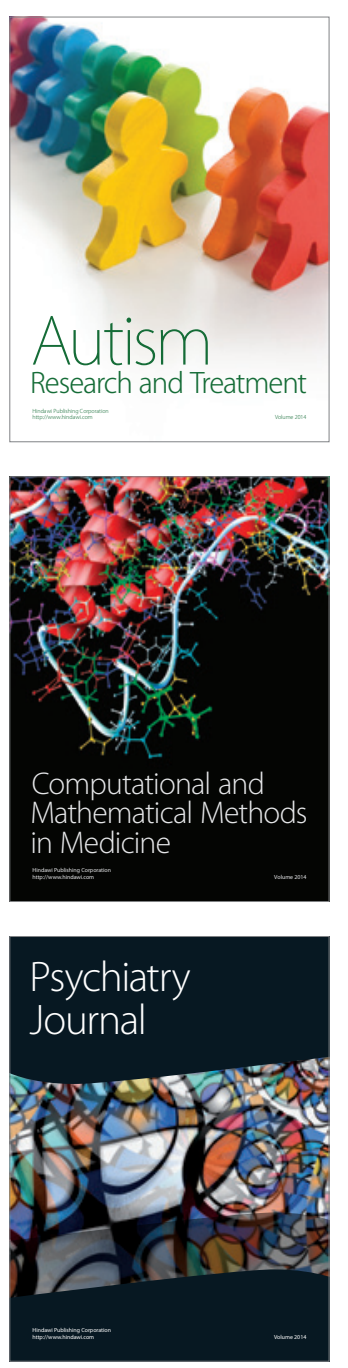
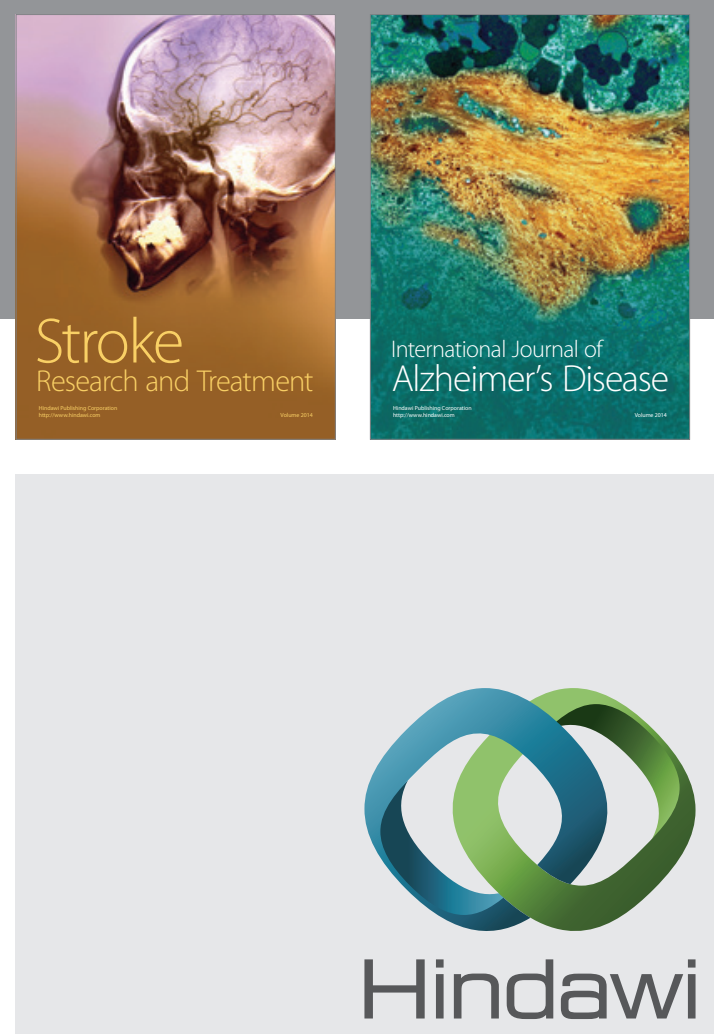

Submit your manuscripts at

http://www.hindawi.com
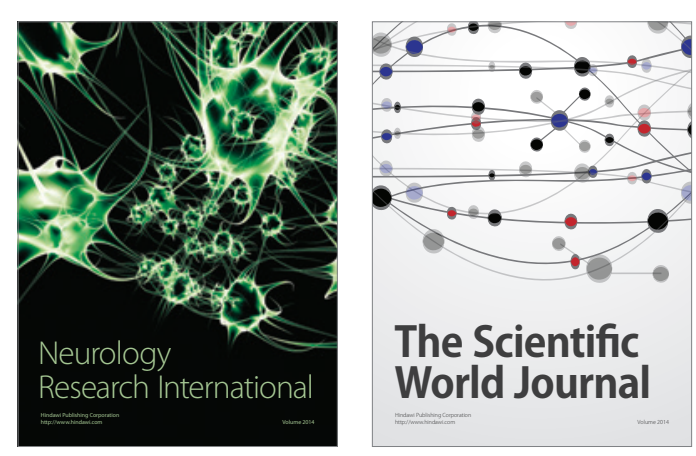

The Scientific World Journal

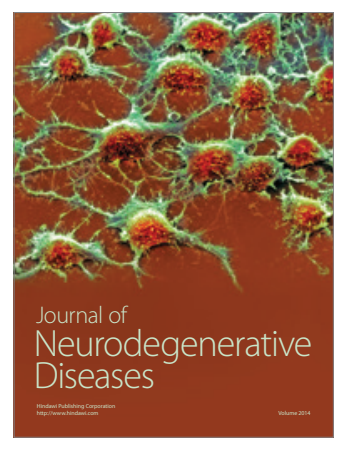

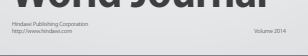

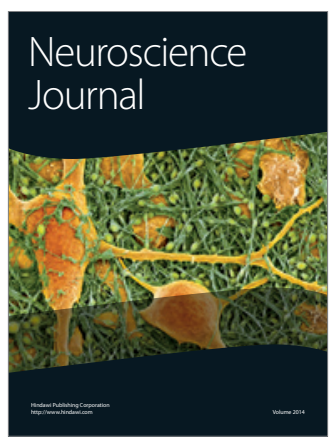

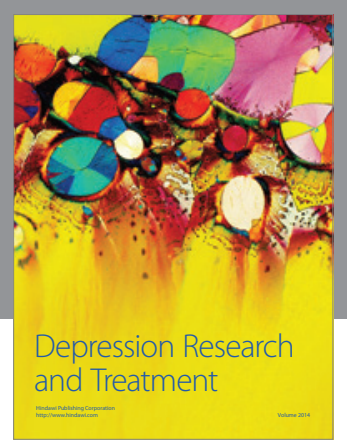
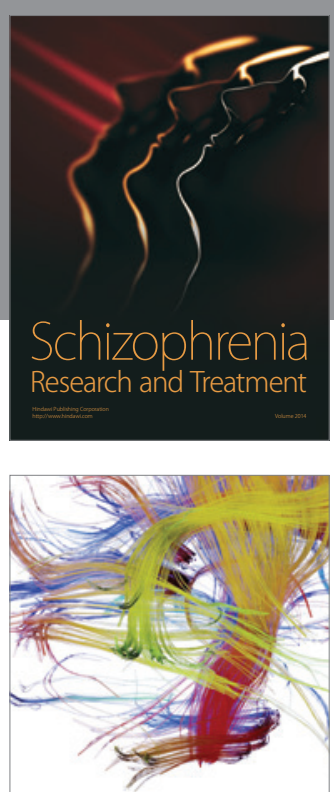

Brain Science

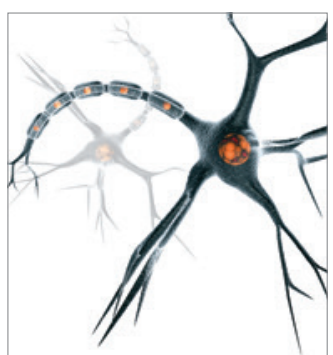

Neural Plasticity
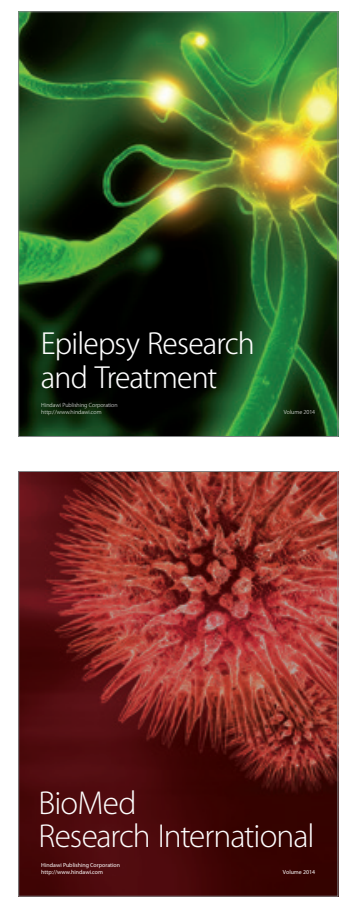

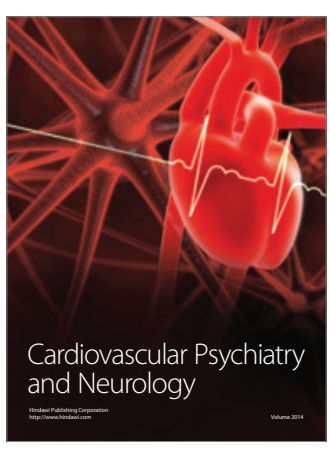

Parkinson's

Disease
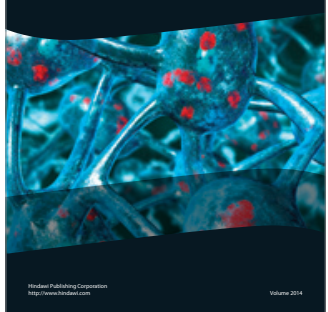